INTERNATIONAL MONETARY FUND

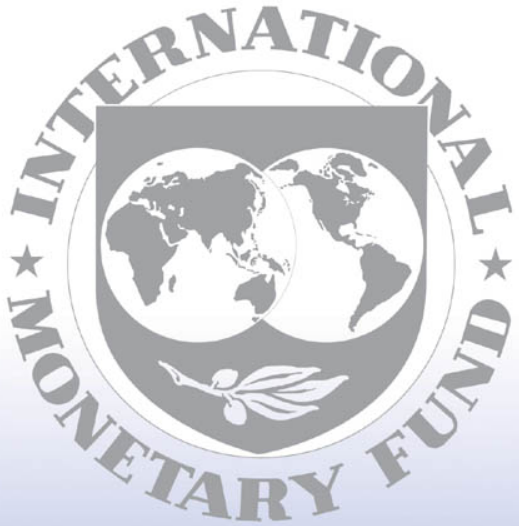

Staff

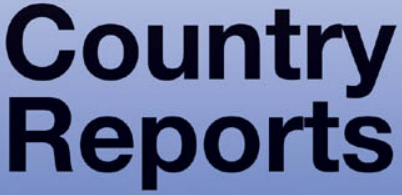




\section{The Federal Democratic Republic of Ethiopia: Statistical Appendix}

This Statistical Appendix for The Federal Democratic Republic of Ethiopia was prepared by a staff team of the International Monetary Fund as background documentation for the periodic consultation with the member country. It is based on the information available at the time it was completed on July 1, 2008. The views expressed in this document are those of the staff team and do not necessarily reflect the views of the government of The Federal Democratic Republic of Ethiopia or the Executive Board of the IMF.

The policy of publication of staff reports and other documents by the IMF allows for the deletion of market-sensitive information.

Copies of this report are available to the public from

International Monetary Fund $\bullet$ Publication Services

700 19th Street, N.W. • Washington, D.C. 20431

Telephone: (202) 623-7430 • Telefax: (202) 623-7201

E-mail: publications@imf.org • Internet: http://www.imf.org

Price: $\$ 18.00$ a copy

\section{International Monetary Fund \\ Washington, D.C.}


This page intentionally left blank 


\section{INTERNATIONAL MONETARY FUND}

\section{THE FEDERAL DEMOCRATIC REPUBLIC OF ETHIOPIA}

\section{Statistical Appendix}

Prepared by the Ethiopian Authorities

(In Conjunction with the IMF Staff Report for the 2008 Article IV Consultation)

Approved by the African Department

July 1,2008

Contents

Page

Tables

Basic Data

1. Gross Domestic Product by Economic Activity at Basic Prices, 2002/03-2006/07 ............. 5

2. Expenditure on Gross Domestic Product at Current Market Prices, 2002/03-2006/07 ......... $\underline{6}$

3. Estimates of Agricultural Production and Cultivated Area of Major Crops, 2002/032006/07

4. Estimates of Coffee Production, Marketing, and Stocks, 2002/03-2006/07 ........................ $\frac{8}{9}$

5. Coffee Prices, July 2005 -December 2007.....................................................................

6. Approval Investment Projects, 2002/03-2006/07 ………................................................10

7. Prices of Petroleum Products, June 2003-January 2008 ………....................................11

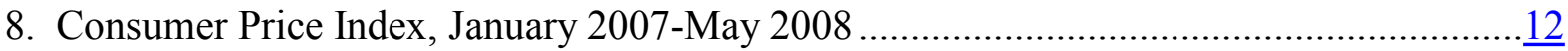

9. Summary of General Government Finances, 2002/03-2006/07 ……................................13

10. General Government Revenues and Grants, 2002/03-2006/07 ......................................14

11. General Government Current Expenditures by Economic Classification,

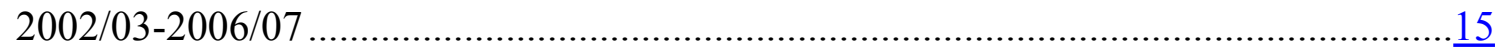

12. General Government Expenditures by Functional Classification, 2002/03-2006/07 .........16

13. Summary of Regional Government Finances, 2002/03-2006/07 ……............................17

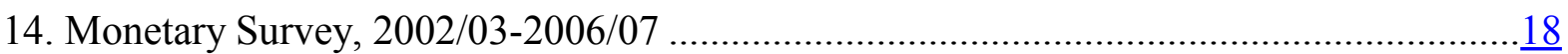

15. Loan Portfolio of the Banking System, 2002/03-2006/07 …………………..................19

16. Sectoral Breakdown of Commercial Bank Claims on Nongovernment Sectors, 2002/03-

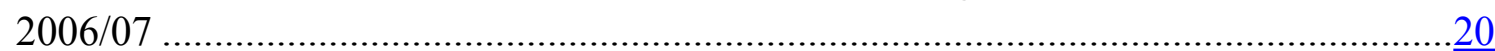

17. Commercial Bank Lending and Deposits, 2002/03-2006/07 …………..........................

18. Reserve and Liquidity Position of Commercial Banks, 2002/03-2006/07 ........................22

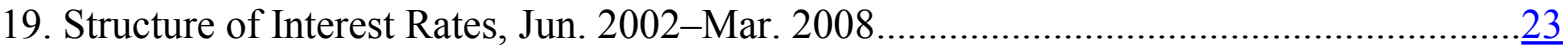

20. Exchange Rate Developments, 2002/03-2006/07 ……..................................................... 


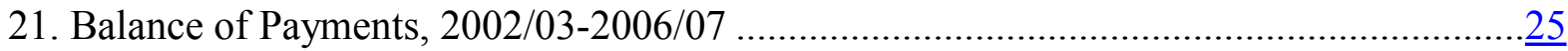

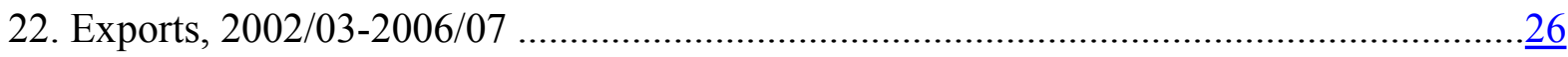

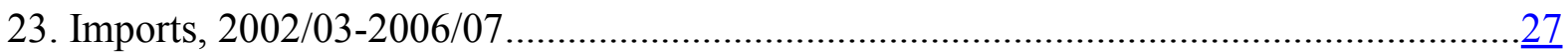

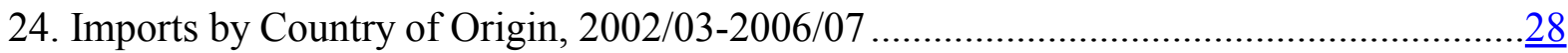

25. Exports by Country of Destination, 2002/03-2006/07 ……………..............................29

26. Public and Publicly Guaranteed External Debt Outstanding, 2002/03-2006/07..................30

27. Financial Soundness Indicators for the Banking Sector, 2003/04-2006/07 ........................31

Summary of the Tax System as of end-June 2008 ……….................................................. 
Ethiopia: Basic Data 1/

Area: $1,221,900$ square kilometers

Population, 2006/07 estimate: 76.1 million

Population, 2001/02-2006/07 average annual growth: 2.82 percent

\begin{tabular}{|c|c|c|c|c|c|}
\hline & $2002 / 03$ & $2003 / 04$ & $2004 / 05$ & $2005 / 06$ & $2006 / 07$ \\
\hline National accounts & \multicolumn{5}{|c|}{ (In millions of birr, unless otherwise specified) } \\
\hline GDP at current basic prices & 68,204 & 79,185 & 98,006 & 121,974 & 158,792 \\
\hline GDP at $1999 / 2000$ basic prices & 66,587 & 74,397 & 83,804 & 93,495 & 104,177 \\
\hline (annual percentage change) & -2.1 & 11.7 & 12.6 & 11.6 & 11.4 \\
\hline \multirow[t]{2}{*}{ GDP deflator (annual percentage change) } & 12.8 & 3.9 & 9.9 & 11.6 & 16.8 \\
\hline & \multicolumn{5}{|c|}{ (In percent of GDP at current market prices) } \\
\hline Gross domestic expenditure & 117.8 & 120.5 & 120.0 & 120.6 & 119.3 \\
\hline Consumption & 96.0 & 95.0 & 97.0 & 96.3 & 94.4 \\
\hline Gross capital formation & 21.8 & 25.5 & 23.0 & 24.2 & 25.0 \\
\hline Net exports & -14.1 & -16.7 & -20.4 & -22.7 & -19.4 \\
\hline \multirow[t]{2}{*}{ Gross domestic savings } & 4.0 & 5.0 & 3.0 & 3.7 & 5.6 \\
\hline & \multicolumn{5}{|c|}{ (Annual percentage change, unless otherwise specified) } \\
\hline \multicolumn{6}{|l|}{ Prices } \\
\hline Consumer price index (annual average) & 15.1 & 8.6 & 6.8 & 15.8 & 17.0 \\
\hline Consumer price index (end of period) & 23.5 & 1.7 & 13.0 & 11.6 & 15.8 \\
\hline \multicolumn{6}{|l|}{ Exchange rates } \\
\hline Ethiopian birr per U.S. dollar (period average) & 8.58 & 8.62 & 8.65 & 8.68 & 8.80 \\
\hline Nominal effective exchange rate (-depreciation; end of period) & -6.4 & -4.6 & -6.9 & -2.7 & -3.9 \\
\hline Real effective exchange rate (- depreciation; end of period) & 14.2 & 3.3 & -6.3 & 8.2 & 12.3 \\
\hline Central government finance & \multicolumn{5}{|c|}{ (In millions of birr, unless otherwise specified) } \\
\hline Total revenue and grants & 15,703 & 17,918 & 20,147 & 24,250 & 30,274 \\
\hline Grants & 4,554 & 4,002 & 4,565 & 4,721 & 8,477 \\
\hline Total expenditure and net lending $2 /$ & 20,504 & 20,504 & 24,796 & 29,325 & 35,608 \\
\hline Recurrent & 13,536 & 11,961 & 13,229 & 15,234 & 17,166 \\
\hline Development and net lending & 6,313 & 8,271 & 11,343 & 14,041 & 18,398 \\
\hline Special program & 655 & 272 & 224 & 50 & 44 \\
\hline \multicolumn{6}{|l|}{ Overall balance $2 /$} \\
\hline (including grants) & $-4,801$ & $-2,586$ & $-4,649$ & $-5,075$ & $-5,334$ \\
\hline (excluding grants) & $-9,355$ & $-6,587$ & $-9,214$ & $-9,796$ & $-13,811$ \\
\hline \multicolumn{6}{|l|}{ Overall balance (in percent of GDP) 2/ } \\
\hline (including grants) & -6.5 & -3.0 & -4.4 & -3.9 & -3.1 \\
\hline (excluding grants) & -12.7 & -7.6 & -8.7 & -7.4 & -8.1 \\
\hline
\end{tabular}


Ethiopia: Basic Data (continued)

\begin{tabular}{|c|c|c|c|c|c|}
\hline & $2002 / 03$ & $2003 / 04$ & $2004 / 05$ & $2005 / 06$ & $2006 / 07$ \\
\hline Money and credit & \multicolumn{5}{|c|}{ (In percent of beginning-period stock of broad money) } \\
\hline Domestic credit $3 /$ & 2.5 & 11.0 & 29.5 & 20.7 & 24.3 \\
\hline Government (net) & 5.7 & 7.1 & 9.3 & 7.1 & 9.0 \\
\hline Other sectors & -3.2 & 3.9 & 20.2 & 13.6 & 15.4 \\
\hline Money and quasi-money (M3) & 10.4 & 10.9 & 19.6 & 17.4 & 19.7 \\
\hline Balance of payments & \multicolumn{5}{|c|}{ (In millions of U.S. dollars, unless otherwise specified) } \\
\hline Current account balance & -109 & -402 & -771 & $-1,387$ & -880 \\
\hline Excluding official transfers (net) & -709 & -969 & $-1,521$ & $-2,253$ & $-2,080$ \\
\hline Exports, f.o.b. & 483 & 600 & 847 & 1,000 & 1,187 \\
\hline Imports, c.i.f. & $-1,856$ & $-2,587$ & $-3,633$ & $-4,593$ & $-5,126$ \\
\hline Trade balance & $-1,374$ & $-1,986$ & $-2,786$ & $-3,592$ & $-3,939$ \\
\hline Services (net) & 167 & 310 & 277 & 149 & 161 \\
\hline Income (net) & -66 & -64 & -36 & -38 & 13 \\
\hline Current transfers (net) & 1,164 & 1,338 & 1,773 & 2,096 & 2,885 \\
\hline Capital and financial account (net) 4/ & 384 & 629 & 675 & 1,037 & 867 \\
\hline Foreign direct investment (net) & 123 & 150 & 150 & 365 & 482 \\
\hline Official long-term loan (net) & 359 & 198 & 271 & 196 & 239 \\
\hline Others & -65 & 164 & 188 & -31 & -24 \\
\hline Overall balance & 275 & 227 & -97 & -350 & -3 \\
\hline \multicolumn{6}{|l|}{ Current account balance (in percent of GDP) } \\
\hline (including official transfers) & -1.3 & -4.0 & -6.3 & -9.1 & -4.5 \\
\hline (excluding official transfers) & -8.3 & -9.6 & -12.4 & -14.9 & -10.6 \\
\hline \multicolumn{6}{|l|}{ Gross official international reserves (end of period) } \\
\hline In millions of U.S. dollars & 930.6 & $1,351.6$ & $1,581.4$ & $1,157.8$ & $1,326.0$ \\
\hline In months of next year's imports & 3.7 & 3.8 & 3.5 & 2.2 & 2.0 \\
\hline External debt & \multicolumn{5}{|c|}{ (In millions of U.S. dollars, unless otherwise specified) } \\
\hline Stock of external debt (end of period) 5/ & 6,738 & 7,371 & 6,021 & 6,005 & 2,292 \\
\hline (in percent of GDP) & 78.7 & 73.3 & 48.9 & 39.6 & 11.8 \\
\hline External debt service & & & & & \\
\hline $\begin{array}{l}\text { (in percent of export of goods and non factor services) } \\
\text { Memorandum item: }\end{array}$ & 7.8 & 6.7 & 5.8 & 5.1 & \\
\hline GDP (In millions of U.S. dollars) & 8,558 & 10,054 & 12,307 & 15,168 & 19,436 \\
\hline
\end{tabular}

Sources: National Bank of Ethiopia, Central Statistical Agency and Ministry of Finance and Economic Development.

$1 /$ Data pertain to the period July 8 -July 7 for the monetary survey and government finance data; and to the period July 1 -June 30 for balance of payments, exchange rate and consumer prices data.

2/ Including special programs.

3/ In percent of beginning-of-period broad money stock.

4 / Including errors and omissions.

5/ Public medium- and long-term debt, including to the Fund. 
Table 1. Ethiopia: Gross Domestic Product by Economic Activity at Basic Prices, 2002/03-2006/07 1/

\begin{tabular}{|c|c|c|c|c|c|}
\hline & $2002 / 03$ & $2003 / 04$ & $2004 / 05$ & $2005 / 06$ & $2006 / 07$ \\
\hline & \multicolumn{5}{|c|}{ (In millions of birr at $1999 / 2000$ prices) } \\
\hline Agriculture and allied activities & 29,920 & 34,990 & 39,729 & 44,063 & 48,226 \\
\hline Industry & 9,331 & 10,420 & 11,402 & 12,561 & 13,943 \\
\hline Mining and quarrying & 346 & 353 & 367 & 394 & 418 \\
\hline Manufacturing & 3,633 & 3,872 & 4,367 & 4,830 & 5,335 \\
\hline Electricity and water & 1,585 & 1,690 & 1,823 & 1,984 & 2,255 \\
\hline Construction & 3,767 & 4,505 & 4,845 & 5,353 & 5,937 \\
\hline Services & 27,799 & 29,536 & 33,312 & 37,769 & 42,876 \\
\hline Distribution services & 13,840 & 14,732 & 16,895 & 19,292 & 22,330 \\
\hline Trade and related services & 9,940 & 10,460 & 11,801 & 13,909 & 16,540 \\
\hline Transportation and communications & 3,900 & 4,272 & 5,094 & 5,384 & 5,790 \\
\hline Other services & 13,959 & 14,804 & 16,417 & 18,477 & 20,546 \\
\hline Banking and insurance & 1,029 & 1,231 & 1,529 & 2,040 & 2,092 \\
\hline Real estate and ownership & 5,785 & 6,045 & 6,492 & 7,424 & 8,538 \\
\hline Public administration and defense & 3,174 & 3,180 & 3,550 & 3,777 & 4,116 \\
\hline Education & 1,891 & 2,108 & 2,373 & 2,578 & 2,935 \\
\hline Health & 606 & 702 & 821 & 860 & 912 \\
\hline Domestic and other & 1,273 & 1,334 & 1,442 & 1,574 & 1,713 \\
\hline Private households with employed persons & 201 & 204 & 210 & 224 & 240 \\
\hline Financial Intermediation Services Indirectly Measured & 463 & 549 & 639 & 898 & 868 \\
\hline Total GDP at $1999 / 2000$ basic prices & 66,587 & 74,397 & 83,804 & 93,495 & 104,177 \\
\hline \multicolumn{6}{|l|}{ Of which } \\
\hline \multirow[t]{2}{*}{ Nonagricultural GDP } & 36,667 & 39,407 & 44,075 & 49,432 & 55,951 \\
\hline & \multicolumn{5}{|c|}{ (Annual percentage change) } \\
\hline Agriculture and allied activities & -10.5 & 16.9 & 13.5 & 10.9 & 9.4 \\
\hline Industry & 6.5 & 11.7 & 9.4 & 10.2 & 11.0 \\
\hline Mining and quarrying & 4.1 & 2.0 & 4.0 & 7.3 & 6.0 \\
\hline Manufacturing & 0.8 & 6.6 & 12.8 & 10.6 & 10.5 \\
\hline Electricity and water & 4.8 & 6.6 & 7.9 & 8.9 & 13.6 \\
\hline Construction & 13.6 & 19.6 & 7.5 & 10.5 & 10.9 \\
\hline Services & 6.0 & 6.2 & 12.8 & 13.4 & 13.5 \\
\hline Distribution services & 5.5 & 6.4 & 14.7 & 14.2 & 15.7 \\
\hline Other services & 6.5 & 6.1 & 10.9 & 12.5 & 11.2 \\
\hline Banking and insurance & 10.9 & 19.6 & 24.2 & 33.4 & 2.5 \\
\hline Real estate and ownership & 9.7 & 4.5 & 7.4 & 14.4 & 15.0 \\
\hline Public administration and defense & 1.4 & 0.2 & 11.6 & 6.4 & 9.0 \\
\hline Education & 11.6 & 11.5 & 12.6 & 8.6 & 13.9 \\
\hline Health & -4.3 & 15.8 & 17.0 & 4.7 & 6.1 \\
\hline Domestic and other & 1.5 & 4.8 & 8.1 & 9.1 & 8.8 \\
\hline Private households with employed persons & 4.7 & 1.5 & 2.9 & 6.8 & 7.0 \\
\hline Total GDP at $1999 / 2000$ basic prices & -2.1 & 11.7 & 12.6 & 11.6 & 11.4 \\
\hline Of which & & & & & \\
\hline Nonagricultural GDP & 6.0 & 7.5 & 11.8 & 12.2 & 13.2 \\
\hline
\end{tabular}

Source: Ministry of Finance and Economic Development.

$1 /$ Data pertain to the period July 8 -July 7. 
Table 2. Ethiopia: Expenditure on Gross Domestic Product at Current Market Prices, 2002/03 - 2006/07 1/

\begin{tabular}{|c|c|c|c|c|c|}
\hline & $2002 / 03$ & $2003 / 04$ & $2004 / 05$ & $2005 / 06$ & $2006 / 07$ \\
\hline & \multicolumn{5}{|c|}{ (In millions of birr) } \\
\hline GDP at current market prices & 73,432 & 86,661 & 106,473 & 131,672 & 170,921 \\
\hline Of which: taxes on products, net & 5,228 & 7,476 & 8,467 & 9,698 & 12,129 \\
\hline Gross domestic expenditure & 86,535 & 104,398 & 127,800 & 158,740 & 203,927 \\
\hline Consumption & 70,495 & 82,328 & 103,317 & 126,846 & 161,277 \\
\hline Private & 60,645 & 71,013 & 90,251 & 110,936 & 143,148 \\
\hline Public & 9,850 & 11,315 & 13,066 & 15,910 & 18,129 \\
\hline Investment & 16,040 & 22,070 & 24,483 & 31,894 & 42,650 \\
\hline Resource gap & $-10,352$ & $-14,453$ & $-21,699$ & $-29,887$ & $-33,225$ \\
\hline Exports of goods and nonfactor services & 9,779 & 12,914 & 16,077 & 18,205 & 21,857 \\
\hline Imports of goods and nonfactor services & 20,131 & 27,367 & 37,776 & 48,092 & 55,082 \\
\hline \multirow[t]{2}{*}{ Statistical Discrepancy } & $-2,751$ & $-3,284$ & 372 & 2,819 & 219 \\
\hline & \multicolumn{5}{|c|}{ (In percent of GDP) } \\
\hline Gross domestic expenditure & 117.8 & 120.5 & 120.0 & 120.6 & 119.3 \\
\hline Consumption & 96.0 & 95.0 & 97.0 & 96.3 & 94.4 \\
\hline Private & 82.6 & 81.9 & 84.8 & 84.3 & 83.8 \\
\hline Public & 13.4 & 13.1 & 12.3 & 12.1 & 10.6 \\
\hline Investment & 21.8 & 25.5 & 23.0 & 24.2 & 25.0 \\
\hline Resource gap & -14.1 & -16.7 & -20.4 & -22.7 & -19.4 \\
\hline Exports of goods and nonfactor services & 13.3 & 14.9 & 15.1 & 13.8 & 12.8 \\
\hline Imports of goods and nonfactor services & 27.4 & 31.6 & 35.5 & 36.5 & 32.2 \\
\hline Statistical Discrepancy & -3.7 & -3.8 & 0.3 & 2.1 & 0.1 \\
\hline Memorandum items: & \multicolumn{5}{|c|}{ (In millions of birr) } \\
\hline GDP at current basic prices & 68,204 & 79,185 & 98,006 & 121,974 & 158,792 \\
\hline GNP at current market prices & 72,865 & 86,113 & 106,163 & 131,338 & 171,036 \\
\hline Gross national income & 82,851 & 97,646 & 121,795 & 149,529 & 196,410 \\
\hline Gross domestic saving & 2,937 & 4,333 & 3,156 & 4,826 & 9,644 \\
\hline Private & 1,638 & 1,731 & 640 & 1,206 & 6,468 \\
\hline Public & 1,299 & 2,602 & 2,516 & 3,620 & 3,176 \\
\hline Gross national saving (including official grants) & 12,356 & 15,318 & 18,478 & 22,683 & 35,133 \\
\hline Private & 6,527 & 8,362 & 9,623 & 12,077 & 22,763 \\
\hline Public & 5,829 & 6,955 & 8,855 & 10,606 & 12,370 \\
\hline
\end{tabular}

Source: Ministry of Finance and Economic Development.

1/ Data pertain to the period July 8-July 7. 
Table 3. Ethiopia: Estimates of Agricultural Production and Cultivated Area of Major Crops, 2002/03-2006/07

\begin{tabular}{|c|c|c|c|c|c|c|c|c|c|c|c|c|c|c|c|}
\hline & \multicolumn{2}{|c|}{$2002 / 03$} & \multirow[b]{2}{*}{ Yield } & \multicolumn{2}{|r|}{ 2003/04 } & \multicolumn{3}{|c|}{ 2004/05 } & \multicolumn{3}{|c|}{ 2005/06 } & \multicolumn{4}{|c|}{$2006 / 07$} \\
\hline & $\begin{array}{r}\text { Cultivated } \\
\text { area }\end{array}$ & $\begin{array}{r}\text { Total } \\
\text { production }\end{array}$ & & $\begin{array}{r}\text { Cultivated } \\
\text { area }\end{array}$ & $\begin{array}{r}\text { Total } \\
\text { productio }\end{array}$ & Yield & $\begin{array}{r}\text { Cultivated } \\
\text { area }\end{array}$ & $\begin{array}{r}\text { Total } \\
\text { production }\end{array}$ & Yield & $\begin{array}{r}\text { Cultivated } \\
\text { area }\end{array}$ & $\begin{array}{r}\text { Total } \\
\text { production }\end{array}$ & Yield & $\begin{array}{r}\text { Cultivated } \\
\text { area }\end{array}$ & $\begin{array}{r}\text { Total } \\
\text { production }\end{array}$ & Yield \\
\hline & \multicolumn{15}{|c|}{ (Area in thousands of hectares; production in thousands of quintals) } \\
\hline Cereals & 6,324 & 63,440 & 10.0 & 6,994 & 90,007 & 12.9 & 7,643 & 100,365 & 13.1 & 8,081 & 116,242 & 14.4 & 8,462 & 128,658 & 15.2 \\
\hline Teff & 1,931 & 14,196 & 7.4 & 1,989 & 16,773 & 8.4 & 2,136 & 20,255 & 9.5 & 2,246 & 21,756 & 9.7 & 2405 & 24377 & 10.1 \\
\hline Barley & 789 & 6,900 & 8.7 & 920 & 10,797 & 11.7 & 1,095 & 13,281 & 12.1 & 998 & 12,707 & 12.7 & 1019 & 13521 & 13.3 \\
\hline Wheat & 998 & 10,721 & 10.7 & 1,099 & 16,144 & 14.7 & 1,398 & 21,766 & 15.6 & 1,460 & 22,191 & 15.2 & 1474 & 24630 & 16.7 \\
\hline Maize & 1,191 & 17,880 & 15.0 & 1,367 & 25,430 & 18.6 & 1,399 & 24,067 & 17.2 & 1,526 & 33,368 & 21.9 & 1694 & 37764 & 22.3 \\
\hline Sorghum & 1,072 & 10,398 & 9.7 & 1,284 & 17,425 & 13.6 & 1,257 & 17,101 & 13.6 & 1,468 & 21,736 & 14.8 & 1464 & 23160 & 15.8 \\
\hline Finger Millet & 308 & 3,092 & 10.0 & 305 & 3,051 & 10.0 & 313 & 3,329 & 10.6 & 333 & 3,970 & 11.9 & 374 & 4844 & 13.0 \\
\hline Oats & 30 & 252 & 8.4 & 30 & 387 & 12.9 & 45 & 567 & 12.6 & 44 & 402 & 9.0 & 32 & 362 & 11.3 \\
\hline Rice & 5 & 0 & - & 0 & 0 & - & 0 & 0 & - & 6 & 112 & 18.0 & & & \\
\hline Pulses & 1,065 & 8,232 & 7.7 & 1,100 & 10,373 & 9.4 & 1,349 & 13,496 & 10.0 & 1,292 & 12,712 & 9.8 & 1,375 & 15,781 & 11.5 \\
\hline Faba beans & 390 & 3,617 & 9.3 & 382 & 4,269 & 11.2 & 463 & 5,520 & 11.9 & 457 & 5,128 & 11.2 & 459 & 5761 & 12.6 \\
\hline Chickpeas & 140 & 1,141 & 8.1 & 154 & 1,359 & 8.8 & 168 & 1,616 & 9.6 & 201 & 2,106 & 10.5 & 200 & 2538 & 12.7 \\
\hline Haricot beans & 185 & 1,117 & 6.0 & 184 & 1,722 & 9.4 & 246 & 2,114 & 8.6 & 164 & 1,384 & 8.5 & 223 & 2227 & 10.0 \\
\hline Field peas & 207 & 1,394 & 6.7 & 212 & 1,704 & 8.1 & 254 & 2,283 & 9.0 & 233 & 1,823 & 7.8 & 221 & 2100 & 9.5 \\
\hline Lentils & 51 & 226 & 4.4 & 52 & 353 & 6.8 & 77 & 546 & 7.1 & 85 & 576 & 6.8 & 97 & 810 & 8.4 \\
\hline Grass peas & 54 & 531 & 9.8 & 83 & 790 & 9.5 & 112 & 1,250 & 11.1 & 123 & 1,459 & 11.8 & 124 & 1837 & 14.8 \\
\hline Fenugreek & 26 & 117 & 4.6 & 17 & 125 & 7.3 & 16 & 116 & 7.1 & 12 & 75 & 6.3 & 20 & 163 & 8.2 \\
\hline Soybeans & 1 & 7 & 7.4 & 1 & 5 & 4.5 & 3 & 8 & 3.2 & 3 & 38 & 11.5 & 6 & 58 & 9.7 \\
\hline Gibto & 11 & 82 & 7.7 & 15 & 48 & 3.2 & 10 & 43 & 4.1 & 14 & 123 & 8.8 & 25 & 287 & 11.5 \\
\hline Oilseeds & 474 & 1,966 & 4.1 & 571 & 3,129 & 5.5 & 825 & 5,266 & 6.4 & 796 & 4,864 & 6.1 & 739 & 4,965 & 6.7 \\
\hline Neug & 250 & 854 & 3.4 & 282 & 1,190 & 4.2 & 359 & 1,872 & 5.2 & 307 & 1,471 & 4.8 & 274 & 1477 & 5.4 \\
\hline Linseed/flax & 132 & 428 & 3.2 & 143 & 774 & 5.4 & 251 & 1,519 & 6.1 & 215 & 1,259 & 5.9 & 174 & 1082 & 6.2 \\
\hline Rapeseed & 17 & 198 & 11.5 & 26 & 293 & 11.3 & 42 & 358 & 8.6 & 25 & 244 & 9.7 & 30 & 292 & 9.7 \\
\hline Sunflower & 4 & 16 & 4.4 & 8 & 50 & 6.0 & 10 & 70 & 7.2 & 9 & 60 & 6.7 & 13 & 111 & 8.5 \\
\hline Groundnuts & 14 & 107 & 7.6 & 20 & 207 & 10.2 & 27 & 291 & 10.7 & 35 & 342 & 9.6 & 37 & 510 & 13.8 \\
\hline Sesame & 58 & 363 & 6.3 & 92 & 615 & 6.7 & 137 & 1,156 & 8.4 & 205 & 1,489 & 7.3 & 211 & 1493 & 7.1 \\
\hline \multirow[t]{2}{*}{ Total } & 7,863 & 73,638 & & 8,664 & 103,509 & & 9,817 & 119,127 & & 10,170 & 133,818 & & 10,576 & 149,404 & \\
\hline & \multicolumn{15}{|c|}{ (Annual percentage change) } \\
\hline Cereals & -0.7 & -27.1 & -26.6 & 10.6 & 41.9 & 28.3 & 9.3 & 11.5 & 2.0 & 5.7 & 15.8 & 9.5 & 4.7 & 10.7 & 5.7 \\
\hline $\begin{array}{l}\text { Pulses } \\
\text { Pula }\end{array}$ & 4.7 & -19.4 & -23.0 & 3.2 & 26.0 & 22.1 & 22.7 & 30.1 & 6.0 & -4.2 & -5.8 & -1.6 & 6.4 & 24.1 & 16.7 \\
\hline Oilseeds & 11.3 & -3.8 & -13.6 & 20.4 & 59.1 & 32.2 & 44.6 & 68.3 & 16.4 & -3.5 & -7.6 & -4.3 & -7.2 & 2.1 & 10.0 \\
\hline Total & 0.6 & -25.9 & - & 10.2 & 40.6 & - & 13.3 & 15.1 & - & 3.6 & 12.3 & - & 4.0 & 11.6 & - \\
\hline
\end{tabular}

Source: Central Statistical Agency. 
Table 4. Ethiopia: Estimates of Coffee Production, Marketing, and Stocks, 2002/03-2006/07 1/

\begin{tabular}{lrrrrr}
\hline & $2002 / 03$ & $2003 / 04$ & $2004 / 05$ & $2005 / 06$ & $2006 / 07$ \\
\hline & \multicolumn{5}{c}{ (In thousands of metric tons) } \\
Opening stocks & 124 & 99 & 79 & 54 & 56 \\
Production & 250 & 260 & 300 & 300 & 321 \\
Domestic consumption & -110 & -110 & -110 & -110 & -110 \\
Exports 2/ & -137 & -142 & -157 & -162 & -166 \\
Unwashed & -99 & -96 & -106 & -116 & -117 \\
Washed & -38 & -46 & -51 & -46 & -49 \\
Adjustments 3/ & -28 & -28 & -58 & -26 & -41 \\
Closing stocks & 99 & 79 & 54 & 56 & 60 \\
\hline
\end{tabular}

Source: Ministry of Agriculture and Rural Development.

1/ Estimates (except exports) based on the findings of the Coffee Sector Survey; coffee year begins in October.

2/ As recorded on loading at port rather than at customs stations.

$3 /$ Represents, among other factors, unofficial exports and handling losses. 
Table 5. Ethiopia: Monthly Coffee Prices, July 2005 -December 2007

\begin{tabular}{|c|c|c|c|c|}
\hline & & $\begin{array}{l}\text { Estimated } \\
\text { Producer } \\
\text { Price 1/ } \\
\end{array}$ & $\begin{array}{l}\text { Auction } \\
\text { Price 2/ }\end{array}$ & \multirow[t]{2}{*}{$\begin{array}{c}\text { ICO } \\
\text { Composite } \\
\text { Price 3/ }\end{array}$} \\
\hline & & \multicolumn{2}{|c|}{ (Birr per metric ton) } & \\
\hline \multirow[t]{12}{*}{$2005 / 06$} & July & 11,049 & 15,968 & 88 \\
\hline & August & 11,611 & 16,612 & 85 \\
\hline & September & 11,096 & 15,858 & 79 \\
\hline & October & 9,675 & 16,111 & 83 \\
\hline & November & 11016 & 16,864 & 87 \\
\hline & December & 11,258 & 17,574 & 89 \\
\hline & January & 12,861 & 19,462 & 101 \\
\hline & February & 11,594 & 17,947 & 97 \\
\hline & March & 11,347 & 17,736 & 93 \\
\hline & April & 10,874 & 17,419 & 94 \\
\hline & May & 10,493 & 16,698 & 90 \\
\hline & June & 9,991 & 15,967 & 86 \\
\hline \multirow[t]{12}{*}{ 2006/07 } & July & 9,537 & 15,579 & 89 \\
\hline & August & 9,533 & 15,751 & 96 \\
\hline & September & 9,510 & 15,745 & 96 \\
\hline & October & 9,435 & 15,589 & 96 \\
\hline & November & 12,764 & 18,792 & 103 \\
\hline & December & 16,290 & 22,701 & 108 \\
\hline & January & 14,846 & 21,329 & 106 \\
\hline & February & 12,720 & 19,094 & 104 \\
\hline & March & 11,200 & 17,712 & 100 \\
\hline & April & 11,485 & 18,101 & 99 \\
\hline & May & 11,946 & 18,874 & 100 \\
\hline & June & 11,936 & 19,156 & 107 \\
\hline \multirow[t]{7}{*}{ 2007/08 } & July & 12,408 & 20,335 & 106 \\
\hline & August & 12,779 & 20,705 & 108 \\
\hline & September & 13,687 & 22,521 & 113 \\
\hline & October & 14,982 & 23,215 & 116 \\
\hline & November & 19,611 & 26,515 & 114 \\
\hline & December & 18,876 & 26,234 & 118 \\
\hline & & \multicolumn{3}{|c|}{ (Annual average, based on coffee year) $4 /$} \\
\hline 2001/02 & & 5,268 & 6,472 & 45 \\
\hline 2002/03 & & 6,277 & 7,778 & 52 \\
\hline 2003/04 & & 8,273 & 10,111 & 58 \\
\hline 2004/05 & & 12,407 & 15,871 & 82 \\
\hline 2005/06 & & 10,641 & 16,904 & 92 \\
\hline \multirow[t]{2}{*}{ 2006/07 } & & 12,625 & 19,576 & 104 \\
\hline & & \multicolumn{3}{|c|}{ (Annual percentage change, based on coffee year) 4/ } \\
\hline 2001/02 & & -40.3 & -36.4 & -5.1 \\
\hline $2002 / 03$ & & 19.2 & 20.2 & 15.3 \\
\hline 2003/04 & & 31.8 & 30.0 & 10.5 \\
\hline 2004/05 & & 50.0 & 57.0 & 41.6 \\
\hline 2005/06 & & -14.2 & 6.5 & 12.2 \\
\hline 2006/07 & & 18.6 & 15.8 & 13.5 \\
\hline
\end{tabular}

Source: Ministry of Agriculture and Rural Development.

$1 /$ Auction price less estimated transport and handling costs.

2/ For unwashed and washed coffee, combined (mostly unwashed at Addis Ababa and weighted by same month's arrival).

$3 /$ ICO is the International Coffee Organization; in U.S. cents per pound.

4/ October-September.

CInternational Monetary Fund. Not for Redistribution 
Table 6. Ethiopia: Approved Investment Projects, 2002/03 - 2006/07 1/

\begin{tabular}{|c|c|c|c|c|c|c|}
\hline & $\begin{array}{c}\begin{array}{c}\text { Number } \\
\text { of } \\
\text { Projects }\end{array} \\
\end{array}$ & $\begin{array}{l}\text { Capital } \\
\text { Cost 2/ }\end{array}$ & $\begin{array}{l}\text { Permanent } \\
\text { Employment }\end{array}$ & $\begin{array}{c}\text { Temporary } \\
\text { Employment }\end{array}$ & $\begin{array}{l}\text { Capital- } \\
\text { Labor } \\
\text { Ratio 3/ } \\
\end{array}$ & $\begin{array}{c}\text { Average } \\
\text { Project } \\
\text { Employment 3/ } \\
\end{array}$ \\
\hline \multicolumn{7}{|l|}{$2002 / 03$} \\
\hline Approved & 1,129 & 12,831 & 47,614 & 20,439 & 0.27 & 42.2 \\
\hline Implementation phase & 70 & 760 & 3,240 & 309 & 0.23 & 46.3 \\
\hline Operational & 142 & 2,098 & 7,366 & 18,850 & 0.28 & 51.9 \\
\hline \multicolumn{7}{|l|}{$2003 / 04$} \\
\hline Approved & 2,157 & 20,564 & 132,964 & 150,109 & 0.15 & 61.6 \\
\hline Implementation phase & 192 & 2,735 & 18,483 & 66,764 & 0.15 & 96.3 \\
\hline Operational & 270 & 3,249 & 18,850 & 22,475 & 0.17 & 69.8 \\
\hline \multicolumn{7}{|l|}{$2004 / 05$} \\
\hline Approved & 3,089 & 38,786 & 137,789 & 612,602 & 0.28 & 44.6 \\
\hline Implementation phase & 175 & 3,277 & 12,693 & 13,353 & 0.26 & 72.5 \\
\hline Operational & 293 & 2,440 & 10,302 & 18,715 & 0.24 & 35.2 \\
\hline \multicolumn{7}{|l|}{$2005 / 06$} \\
\hline Approved & 5,811 & 79,773 & 209,592 & 335,740 & 0.38 & 36.1 \\
\hline Implementation phase & 42 & 295 & 1,433 & 3,684 & 0.21 & 34.1 \\
\hline Operational & 203 & 802 & 3,949 & 5,567 & 0.20 & 19.5 \\
\hline \multicolumn{7}{|l|}{$2006 / 07$} \\
\hline Approved & 6,899 & 101,284 & 378,050 & 749,523 & 0.27 & 54.8 \\
\hline Implementation phase & 58 & 652 & 2,553 & 5,394 & 0.26 & 44.0 \\
\hline Operational & 193 & 1,698 & 8,653 & 20,931 & 0.20 & 44.8 \\
\hline
\end{tabular}

Source: Ethiopian Investment Agency.

1/ Excludes projects with initial investment of less than $\mathrm{Br} 250,000$.

2/ As stated in the project application; in thousands of birr.

3/ Capital - labor ratio and average employment per project are calculated using permanent employment. 
Table 7. Ethiopia: Prices of Petroleum Products, June 2003-January 2008 1/

\begin{tabular}{|c|c|c|c|c|}
\hline & $\begin{array}{l}\text { Regular } \\
\text { Gasoline }\end{array}$ & $\begin{array}{c}\text { Gas Oil } \\
\text { (Diesel) }\end{array}$ & $\begin{array}{c}\text { Fuel } \\
\text { Oil }\end{array}$ & $\begin{array}{l}\text { Lighting } \\
\text { Kerosene }\end{array}$ \\
\hline & \multicolumn{4}{|c|}{ (In birr per liter, unless otherwise indicated) } \\
\hline \multicolumn{5}{|l|}{ June 2003 - October 2003} \\
\hline Import border price & 1.92 & 1.82 & 1.57 & 1.75 \\
\hline Addis Ababa & 4.40 & 2.72 & 2.30 & 2.00 \\
\hline (in percentage change) & 0.00 & 0.00 & 0.00 & 0.00 \\
\hline (in U.S. dollars per liter) & 0.51 & 0.32 & 0.27 & 0.23 \\
\hline \multicolumn{5}{|l|}{ November 2003 - March 2004} \\
\hline Import border price & 1.77 & 1.85 & 1.64 & 1.87 \\
\hline Addis Ababa & 4.40 & 2.72 & 2.30 & 2.00 \\
\hline (in percentage change) & 0.00 & 0.00 & 0.00 & 0.00 \\
\hline (in U.S. dollars per liter) & 0.51 & 0.32 & 0.27 & 0.23 \\
\hline \multicolumn{5}{|l|}{ April 2004 - August 2004} \\
\hline Import border price & 2.27 & 2.16 & 1.65 & 2.17 \\
\hline Addis Ababa & 4.69 & 3.02 & 2.56 & 2.15 \\
\hline (in percentage change) & 6.59 & 11.03 & 11.30 & 7.50 \\
\hline (in U.S. dollars per liter) & 0.54 & 0.35 & 0.30 & 0.25 \\
\hline \multicolumn{5}{|c|}{ September 2004 - November 2004} \\
\hline Import border price & 2.75 & 2.66 & 1.81 & 2.81 \\
\hline Addis Ababa & 5.12 & 3.63 & 3.17 & 2.69 \\
\hline (in percentage change) & 9.17 & 20.20 & 23.83 & 25.12 \\
\hline (in U.S. dollars per liter) & 0.59 & 0.42 & 0.37 & 0.31 \\
\hline \multicolumn{5}{|l|}{ December 2004 - May 2006} \\
\hline Import border price & 2.93 & 3.21 & 1.88 & 3.45 \\
\hline Addis Ababa & 5.50 & 4.31 & 3.52 & 3.00 \\
\hline (in percentage change) & 7.42 & 18.73 & 11.04 & 11.52 \\
\hline (in U.S. dollars per liter) & 0.64 & 0.50 & 0.41 & 0.35 \\
\hline \multicolumn{5}{|l|}{ May 2006 - August 2006} \\
\hline Import border price & 3.96 & 4.48 & 3.22 & 4.63 \\
\hline Addis Ababa & 6.58 & 4.78 & 4.19 & 3.45 \\
\hline (in percentage change) & 19.60 & 10.90 & 19.00 & 15.00 \\
\hline (in U.S. dollars per liter) & 0.75 & 0.55 & 0.48 & 0.40 \\
\hline \multicolumn{5}{|l|}{ August 2006 - January 2007} \\
\hline Import border price & 4.85 & 5.08 & 3.20 & 5.23 \\
\hline Addis Ababa & 8.17 & 5.44 & 4.19 & 4.12 \\
\hline (in percentage change) & 24.16 & 13.81 & 0.00 & 19.42 \\
\hline (in U.S. dollars per liter) & 0.94 & 0.63 & 0.48 & 0.47 \\
\hline \multicolumn{5}{|l|}{ January 2007-December 2007} \\
\hline Import border price & 3.53 & 4.20 & 2.67 & 4.59 \\
\hline Addis Ababa & 7.77 & 5.44 & 4.19 & 4.12 \\
\hline (in percentage change) & -4.90 & 0.00 & 0.00 & 0.00 \\
\hline (in U.S. dollars per liter) & 0.88 & 0.62 & 0.48 & 0.47 \\
\hline \multicolumn{5}{|l|}{ January 2008- } \\
\hline Import border price & 6.65 & 6.80 & 4.81 & 6.90 \\
\hline Addis Ababa & 9.61 & 6.90 & 5.90 & 5.72 \\
\hline (in percentage change) & 23.68 & 26.84 & 40.81 & 38.83 \\
\hline (in U.S. dollars per liter) & 1.04 & 0.75 & 0.64 & 0.62 \\
\hline
\end{tabular}

1/ Retail prices vary, depending on transportation costs. 
Table 8. Ethiopia: Consumer Price Index, January 2007-May 2008

\begin{tabular}{|c|c|c|c|c|c|c|c|}
\hline & & \multicolumn{3}{|c|}{ National } & \multicolumn{3}{|c|}{ Addis Ababa } \\
\hline & & Overall & Food & Nonfood & General & Food & Nonfood \\
\hline & & \multicolumn{6}{|c|}{$($ December $2006=100)$} \\
\hline \multirow[t]{12}{*}{2007} & January & 100.4 & 100.0 & 100.9 & 100.5 & 100.5 & 100.5 \\
\hline & February & 102.3 & 102.9 & 101.5 & 101.8 & 102.4 & 101.3 \\
\hline & March & 104.3 & 105.4 & 102.9 & 103.6 & 107.9 & 100.6 \\
\hline & April & 105.8 & 107.7 & 103.3 & 105.1 & 110.2 & 101.6 \\
\hline & May & 106.3 & 107.9 & 104.1 & 107.1 & 113.2 & 102.8 \\
\hline & June & 107.4 & 109.5 & 104.5 & 107.3 & 111.9 & 104.1 \\
\hline & July & 109.6 & 113.0 & 105.1 & 109.3 & 116.3 & 104.5 \\
\hline & August & 112.7 & 118.1 & 105.6 & 111.2 & 120.9 & 104.5 \\
\hline & September & 115.3 & 121.9 & 106.5 & 113.2 & 126.0 & 104.3 \\
\hline & October & 117.1 & 124.4 & 107.5 & 113.0 & 124.6 & 105.0 \\
\hline & November & 117.2 & 123.8 & 108.5 & 111.8 & 118.1 & 107.4 \\
\hline & December & 118.4 & 125.7 & 108.8 & 115.3 & 123.2 & 109.7 \\
\hline \multirow[t]{6}{*}{2008} & January & 120.2 & 127.9 & 109.9 & 116.0 & 124.3 & 110.2 \\
\hline & February & 125.7 & 133.9 & 114.9 & 122.2 & 131.1 & 116.0 \\
\hline & March & 135.1 & 146.9 & 119.6 & 129.7 & 142.6 & 120.8 \\
\hline & April & 137.1 & 149.7 & 120.3 & 130.2 & 143.2 & 121.2 \\
\hline & May & 147.8 & 166.3 & 123.3 & 140.8 & 165.2 & 123.8 \\
\hline & & \multicolumn{6}{|c|}{ (Annual percentage change; end of period) } \\
\hline \multirow[t]{12}{*}{2007} & January & 16.6 & 17.8 & 14.8 & 21.0 & 26.5 & 15.7 \\
\hline & February & 17.5 & 20.0 & 13.9 & 19.6 & 25.1 & 14.6 \\
\hline & March & 18.6 & 21.5 & 14.4 & 21.1 & 30.0 & 14.0 \\
\hline & April & 20.0 & 23.7 & 15.0 & 22.4 & 31.0 & 15.5 \\
\hline & May & 16.0 & 18.8 & 12.2 & 21.5 & 31.8 & 13.9 \\
\hline & June & 15.1 & 18.2 & 10.9 & 17.3 & 24.1 & 12.2 \\
\hline & July & 14.3 & 18.3 & 8.8 & 17.7 & 27.3 & 10.9 \\
\hline & August & 16.0 & 21.9 & 8.1 & 17.8 & 29.6 & 9.5 \\
\hline & September & 17.7 & 25.0 & 7.9 & 15.4 & 31.2 & 4.4 \\
\hline & October & 18.7 & 26.1 & 8.8 & 13.5 & 25.8 & 5.0 \\
\hline & November & 18.1 & 24.8 & 9.2 & 12.3 & 19.7 & 7.3 \\
\hline & December & 18.4 & 25.6 & 8.8 & 15.3 & 23.2 & 9.7 \\
\hline \multirow[t]{6}{*}{2008} & January & 19.7 & 27.9 & 9.0 & 15.4 & 23.7 & 9.6 \\
\hline & February & 22.9 & 30.2 & 13.2 & 20.1 & 28.0 & 14.5 \\
\hline & March & 29.6 & 39.4 & 16.2 & 25.2 & 32.2 & 20.0 \\
\hline & April & 29.6 & 39.1 & 16.5 & 23.8 & 30.0 & 19.3 \\
\hline & May & 39.1 & 54.1 & 18.5 & 31.5 & 45.9 & 20.4 \\
\hline & & \multicolumn{6}{|c|}{ (Annual percentage change; annual average) } \\
\hline 2007 & January & 12.9 & 14.0 & 11.3 & 15.2 & 20.7 & 10.4 \\
\hline & February & 13.5 & 14.5 & 12.0 & 16.0 & 21.4 & 11.2 \\
\hline & March & 14.2 & 15.3 & 12.7 & 16.9 & 22.5 & 12.0 \\
\hline & June & 15.8 & 17.5 & 13.5 & 19.2 & 25.4 & 14.0 \\
\hline & July & 15.9 & 17.9 & 13.2 & 19.5 & 26.1 & 14.1 \\
\hline & August & 16.1 & 18.6 & 12.8 & 19.8 & 27.0 & 14.0 \\
\hline & September & 16.5 & 19.6 & 12.3 & 19.5 & 27.8 & 13.0 \\
\hline & October & 16.9 & 20.6 & 11.9 & 18.9 & 27.9 & 12.1 \\
\hline & November & 17.1 & 21.3 & 11.5 & 18.2 & 27.3 & 11.3 \\
\hline & December & 17.2 & 21.9 & 11.0 & 17.7 & 27.0 & 10.9 \\
\hline 2008 & January & 17.5 & 22.8 & 10.5 & 17.3 & 26.7 & 10.4 \\
\hline & February & 18.0 & 23.6 & 10.5 & 17.3 & 27.0 & 10.4 \\
\hline & March & 19.0 & 25.2 & 10.7 & 17.8 & 27.2 & 11.0 \\
\hline & April & 19.8 & 26.6 & 10.8 & 18.0 & 27.2 & 11.4 \\
\hline & May & 22.0 & 29.7 & 11.2 & 18.9 & 28.7 & 11.9 \\
\hline & & & & (Mor & change) & & \\
\hline 2007 & January & 0.4 & 0.0 & 0.9 & 0.5 & 0.5 & 0.5 \\
\hline & February & 1.9 & 2.9 & 0.6 & 1.2 & 1.9 & 0.8 \\
\hline & March & 2.0 & 2.5 & 1.4 & 1.8 & 5.3 & -0.7 \\
\hline & April & 1.4 & 2.1 & 0.4 & 1.5 & 2.1 & 1.0 \\
\hline & May & 0.4 & 0.2 & 0.8 & 1.9 & 2.8 & 1.2 \\
\hline & June & 1.0 & 1.5 & 0.4 & 0.2 & -1.2 & 1.2 \\
\hline & July & 2.1 & 3.1 & 0.6 & 1.9 & 3.9 & 0.3 \\
\hline & August & 2.9 & 4.5 & 0.5 & 1.8 & 4.0 & 0.0 \\
\hline & September & 2.3 & 3.3 & 0.8 & 1.8 & 4.2 & -0.2 \\
\hline & October & 1.6 & 2.0 & 1.0 & -0.2 & -1.1 & 0.7 \\
\hline & November & 0.1 & -0.5 & 0.9 & -1.1 & -5.2 & 2.3 \\
\hline & December & 1.0 & 1.5 & 0.3 & 3.1 & 4.3 & 2.2 \\
\hline 2008 & January & 1.5 & 1.8 & 1.1 & 0.6 & 0.9 & 0.4 \\
\hline & February & 4.6 & 4.7 & 4.5 & 5.4 & 5.5 & 5.3 \\
\hline & March & 7.5 & 9.7 & 4.2 & 6.2 & 8.8 & 4.1 \\
\hline & April & 1.4 & 1.9 & 0.6 & 0.4 & 0.4 & 0.4 \\
\hline & May & 7.8 & 11.1 & 2.5 & 8.1 & 15.4 & 2.1 \\
\hline
\end{tabular}

Sources: Central Statistical Agency, and National Bank of Ethiopia. 
Table 9. Ethiopia: Summary of General Government Finances, 2002/03-2006/07 1/

\begin{tabular}{|c|c|c|c|c|c|}
\hline & $2002 / 03$ & $2003 / 04$ & $2004 / 05$ & $2005 / 06$ & $2006 / 07$ \\
\hline & \multicolumn{5}{|c|}{ (In millions of birr) } \\
\hline Total revenue and grants & 15,703 & 17,918 & 20,147 & $24,250.0$ & 30,274 \\
\hline Revenue & 11,150 & 13,917 & 15,582 & $19,529.4$ & 21,797 \\
\hline Tax revenue & 8,243 & 10,906 & 12,398 & 14,159 & 17,354 \\
\hline Nontax revenue & 2,907 & 3,010 & 3,184 & 5,371 & 4,443 \\
\hline Grants & 4,554 & 4,002 & 4,565 & $4,720.7$ & 8,477 \\
\hline Total expenditure and net lending (cash basis) & 20,504 & 20,504 & 24,796 & 29,325 & 35,608 \\
\hline Recurrent expenditure & 13,536 & 11,961 & 13,229 & $15,233.8$ & $17,165.9$ \\
\hline Capital expenditure & 6,313 & 8,271 & 11,343 & $14,041.4$ & $18,398.0$ \\
\hline Special programs & 655 & 272 & 224 & 50 & 44 \\
\hline \multicolumn{6}{|l|}{ Overall balance } \\
\hline Including grants & $-4,801$ & $-2,586$ & $-4,649$ & $-5,075.2$ & $-5,334$ \\
\hline Excluding grants & $-9,355$ & $-6,587$ & $-9,214$ & $-9,796$ & $-13,811$ \\
\hline Financing & 4,801 & 2,586 & 4,649 & $5,075.2$ & 5,334 \\
\hline Net external financing & 3,865 & 2,399 & 2,384 & $1,512.3$ & 1,916 \\
\hline Net domestic financing & 1,653 & 2,147 & 3,492 & $1,746.4$ & 5,357 \\
\hline Banking system & 1,651 & 2,155 & 3,156 & $2,876.1$ & 3,369 \\
\hline Nonbank sources & 2 & -8 & 336 & -140.7 & 1,988 \\
\hline MDRI, account held at NBE & 0 & 0 & 0 & -989 & 0 \\
\hline Privatization & 8 & 11 & 10 & 0 & 0 \\
\hline \multirow[t]{2}{*}{ Float/unidentified financing } & -725 & $-1,971$ & $-1,237$ & 1,816 & $-1,939$ \\
\hline & \multicolumn{5}{|c|}{ (In Percent of GDP) } \\
\hline Total revenue and grants & 21.4 & 20.7 & 18.9 & 18.4 & 17.7 \\
\hline Revenue & 15.2 & 16.1 & 14.6 & 14.8 & 12.8 \\
\hline Tax revenue & 11.2 & 12.6 & 11.6 & 10.8 & 10.2 \\
\hline Nontax revenue & 4.0 & 3.5 & 3.0 & 4.1 & 2.6 \\
\hline Grants & 6.2 & 4.6 & 4.3 & 3.6 & 5.0 \\
\hline Total expenditure and net lending (cash basis) & 27.9 & 23.7 & 23.3 & 22.3 & 20.8 \\
\hline Recurrent expenditure & 18.4 & 13.8 & 12.4 & 11.6 & 10.0 \\
\hline Capital expenditure & 8.6 & 9.5 & 10.7 & 10.7 & 10.8 \\
\hline Special programs & 0.9 & 0.3 & 0.2 & 0.0 & 0.0 \\
\hline \multicolumn{6}{|l|}{ Overall balance } \\
\hline Including grants & -6.5 & -3.0 & -4.4 & -3.9 & -3.1 \\
\hline Excluding grants & -12.7 & -7.6 & -8.7 & -7.4 & -8.1 \\
\hline Financing & 6.5 & 3.0 & 4.4 & 3.9 & 3.1 \\
\hline Net external financing & 5.3 & 2.8 & 2.2 & 1.1 & 1.1 \\
\hline Domestic (net) & 2.3 & 2.5 & 3.3 & 1.3 & 3.1 \\
\hline Banking system & 2.2 & 2.5 & 3.0 & 2.2 & 2.0 \\
\hline Nonbank sources & 0.0 & 0.0 & 0.3 & -0.1 & 1.2 \\
\hline Privatization & 0.0 & 0.0 & 0.0 & 0.0 & 0.0 \\
\hline Float/unidentified financing & -1.0 & -2.3 & -1.2 & 1.4 & -1.1 \\
\hline
\end{tabular}

Source: Ministry of Finance and Economic Development.

$1 /$ Ethiopian fiscal year ending July 7 . 
Table 10. Ethiopia: General Government Revenues and Grants, 2002/03-2006/07 $1 /$

\begin{tabular}{|c|c|c|c|c|c|}
\hline & $2002 / 03$ & $2003 / 04$ & $2004 / 05$ & $2005 / 06$ & $2006 / 07$ \\
\hline & \multicolumn{5}{|c|}{ (In million of Birr) } \\
\hline Tax revenue & 8,243 & 10,906 & 12,398 & 14,159 & 17,354 \\
\hline Direct taxes & 3,010 & 3,431 & 3,930 & 4,461 & 5,168 \\
\hline Income and profits tax & 2,878 & 3,131 & 3,569 & 3,819 & 4,868 \\
\hline Personal income & 833 & 948 & 1,132 & 1,414 & 1,828 \\
\hline Rental income tax & 52 & 53 & 78 & 32 & 26 \\
\hline Business profits & 1,639 & 1,303 & 1,714 & 1,741 & 2,305 \\
\hline Withholding income tax on imports & 228 & 448 & 415 & 407 & 433 \\
\hline Agriculture income & 72 & 138 & 150 & 82 & 98 \\
\hline Other income & 24 & 204 & 37 & 86 & 110 \\
\hline Interest income tax & 21 & 20 & 23 & 29 & 52 \\
\hline Capital gains tax & 10 & 17 & 20 & 29 & 16 \\
\hline Rural land use fee & 89 & 114 & 140 & 124 & 130 \\
\hline Urban land lease fee & 44 & 186 & 221 & 517 & 170 \\
\hline Indirect taxes & 5,233 & 7,476 & 8,468 & 9,698 & 12,186 \\
\hline Domestic indirect taxes & 1,668 & 2,200 & 2,721 & 3,111 & 3,997 \\
\hline Sales/excise taxes & 1,144 & 1,534 & 2,003 & 2,301 & 2,863 \\
\hline Petroleum products & & & & 0 & 0 \\
\hline Alcohol and tobacco & 278 & 279 & 322 & 442 & 528 \\
\hline Other goods & 866 & 1,255 & 1,681 & 1,859 & 2,335 \\
\hline Services sales tax & 397 & 505 & 533 & 597 & 856 \\
\hline Stamp duties & 127 & 161 & 185 & 213 & 278 \\
\hline Import duties and taxes & 3,564 & 5,276 & 5,746 & 6,587 & 8,189 \\
\hline Custom duties & 1,606 & 2,434 & 2,503 & 2,954 & 3,298 \\
\hline Sales/excise taxes & 1,888 & 2,833 & 3,242 & 3,633 & 4,398 \\
\hline Surtax on imports & 70 & 10 & 1 & 0 & 492 \\
\hline Export taxes & 1 & 0 & 0 & 0 & 0 \\
\hline Non-tax revenue & 2,907 & 3,010 & 3,184 & 5,371 & 4,443 \\
\hline Charges and fees & 252 & 321 & 616 & 379 & 342 \\
\hline Sales of goods \& services & 330 & 376 & 856 & 433 & 250 \\
\hline \multicolumn{6}{|l|}{ Residual surplus, capital charge, interest } \\
\hline payments and state dividend & 1,102 & 1,250 & 1,106 & 3,136 & 2,682 \\
\hline Pension contributions & -- & -- & -- & 0 & -- \\
\hline Reimbursement \& property sales & 204 & 185 & 193 & 310 & 168 \\
\hline Miscellaneous & 484 & 716 & 400 & 172 & 327 \\
\hline Other revenue & 535 & 163 & 14 & 941 & 674 \\
\hline Privatization proceeds (below the line for IMF tables) & 0 & 0 & & 0 & \\
\hline Total revenue & 11,150 & 13,917 & 15,582 & 19,529 & 21,797 \\
\hline External grants & 4,554 & 4,002 & 4,565 & 4,721 & 8,477 \\
\hline Grants in kind/earmarked & 4,087 & 1,746 & 2,234 & 2,782 & 3,492 \\
\hline Untied cash \& CPF/grants & 467 & 2,255 & 2,331 & 950 & 4,985 \\
\hline Multilateral Debt Relief Initiative & 0 & 0 & 0 & 989 & 0 \\
\hline \multirow[t]{2}{*}{ Total revenue and grants } & 15,703 & 17,918 & 20,147 & 24,250 & 30,274 \\
\hline & \multicolumn{5}{|c|}{ (In percent of total revenue) } \\
\hline Tax revenue & 73.9 & 78.4 & 79.6 & 72.5 & 79.6 \\
\hline Direct taxes & 27.0 & 24.7 & 25.2 & 22.8 & 23.7 \\
\hline Indirect taxes & 46.9 & 53.7 & 54.3 & 49.7 & 55.9 \\
\hline \multirow[t]{2}{*}{ Non-tax revenue } & \multirow{2}{*}{\multicolumn{5}{|c|}{ (In percent of GDP) }} \\
\hline & & & & & \\
\hline Tax revenue & 11.2 & 12.6 & 11.6 & 10.8 & 10.2 \\
\hline Direct taxes & 4.1 & 4.0 & 3.7 & 3.4 & 3.0 \\
\hline Income and profits tax & 3.9 & 3.6 & 3.4 & 2.9 & 2.8 \\
\hline Rural land use fee & 0.1 & 0.1 & 0.1 & 0.1 & 0.1 \\
\hline Indirect taxes & 7.1 & 8.6 & 8.0 & 7.4 & 7.1 \\
\hline Domestic indirect taxes & 2.3 & 2.5 & 2.6 & 2.4 & 2.3 \\
\hline Import duties and taxes & 4.9 & 6.1 & 5.4 & 5.0 & 4.8 \\
\hline Export taxes & 0.0 & 0.0 & 0.0 & 0.0 & 0.0 \\
\hline Non-tax revenue & 4.0 & 3.5 & 3.0 & 4.1 & 2.6 \\
\hline Residual surplus, capital charge, interest & 1.5 & 1.4 & 1.0 & 2.4 & 1.6 \\
\hline Other extraordinary & 0.7 & 0.2 & 0.0 & 0.7 & 0.4 \\
\hline Grants & 6.2 & 4.6 & 4.3 & 3.6 & 5.0 \\
\hline
\end{tabular}

Source: Ministry of Finance and Economic Development.

$1 /$ Ethiopian fiscal year ending July 7. 
Table 11. Ethiopia: General Government Current Expenditures by Economic Classification, 2002/03-2006/07 1/

\begin{tabular}{|c|c|c|c|c|c|}
\hline & $2002 / 03$ & $2003 / 04$ & $2004 / 05$ & $2005 / 06$ & $2006 / 07$ \\
\hline & \multicolumn{5}{|c|}{ (In millions of birr) } \\
\hline Wages and operating expenses & 8,764 & 9,607 & 10,777 & 12,876 & 14,838 \\
\hline Wages and salaries & 4,001 & 5,162 & 5,949 & 7,995 & 9,349 \\
\hline Materials & 4,762 & 4,445 & 4,828 & 4,881 & 5,489 \\
\hline Grants and contributions & 410 & 346 & 392 & 294 & 271 \\
\hline Pensions & 254 & 234 & 328 & 425 & 438 \\
\hline Interest and charges & 1,219 & 1,080 & 1,011 & 1,054 & 1,207 \\
\hline Domestic debt & 625 & 576 & 525 & 621 & 727 \\
\hline External debt & 594 & 504 & 486 & 433 & 480 \\
\hline External assistance & 2,890 & 699 & 721 & 586 & 411 \\
\hline \multirow[t]{2}{*}{ Total current expenditure } & 13,536 & 11,961 & 13,229 & 15,234 & 17,166 \\
\hline & \multicolumn{5}{|c|}{ (In percent of total current expenditure) } \\
\hline Wages and operating expenses & 64.7 & 80.3 & 81.5 & 84.5 & 86.4 \\
\hline Wages and salaries & 29.6 & 43.2 & 45.0 & 52.5 & 54.5 \\
\hline Materials & 35.2 & 37.2 & 36.5 & 32.0 & 32.0 \\
\hline Grants and contributions & 3.0 & 2.9 & 3.0 & 1.9 & 1.6 \\
\hline Subsidies & 0.0 & 0.0 & 0.0 & 0.0 & 0.0 \\
\hline Pensions & 1.9 & 2.0 & 2.5 & 2.8 & 2.6 \\
\hline Interest and charges & 9.0 & 9.0 & 7.6 & 6.9 & 7.0 \\
\hline \multirow[t]{2}{*}{ External assistance } & 21.4 & 5.8 & 5.5 & 3.8 & 2.4 \\
\hline & \multicolumn{5}{|c|}{ (In percent of GDP) } \\
\hline Wages and operating expenses & 11.9 & 11.1 & 10.1 & 9.8 & 8.7 \\
\hline Wages and salaries & 5.4 & 6.0 & 5.6 & 6.1 & 5.5 \\
\hline Materials & 6.5 & 5.1 & 4.5 & 3.7 & 3.2 \\
\hline Grants and contributions & 0.6 & 0.4 & 0.4 & 0.2 & 0.2 \\
\hline Subsidies & 0.0 & 0.0 & 0.0 & 0.0 & 0.0 \\
\hline Pensions & 0.3 & 0.3 & 0.3 & 0.3 & 0.3 \\
\hline Interest and charges & 1.7 & 1.2 & 0.9 & 0.8 & 0.7 \\
\hline External assistance & 3.9 & 0.8 & 0.7 & 0.4 & 0.2 \\
\hline Total current expenditure & 18.4 & 13.8 & 12.4 & 11.6 & 10.0 \\
\hline
\end{tabular}

Source: Ministry of Finance and Economic Development.

$1 /$ Ethiopian fiscal year ending July 7. 


\begin{tabular}{|c|c|c|c|c|c|}
\hline & $2002 / 03$ & $2003 / 04$ & $2004 / 05$ & $2005 / 06$ & $2006 / 07$ \\
\hline & \multicolumn{5}{|c|}{ (In millions of birr) } \\
\hline Current expenditure & 13,536 & 11,961 & 13,229 & 15,234 & 17,166 \\
\hline General services & 4,679 & 5,044 & 5,816 & 6,522 & 7,073 \\
\hline Defense & 2,341 & 2,452 & 2,920 & 3,009 & 3,005 \\
\hline Public order and security & 792 & 766 & 892 & 1,201 & 1,362 \\
\hline General services 2/ & 1,545 & 1,826 & 2,004 & 2,311 & 2,706 \\
\hline Economic services & 1,337 & 1,361 & 1,516 & 2,009 & 2,201 \\
\hline Agriculture and natural resources & 730 & 870 & 1,158 & 1,566 & 1,703 \\
\hline Trade, Industry and tourism & 235 & 125 & 117 & 152 & 177 \\
\hline Mines and energy & 47 & 44 & 26 & 38 & 41 \\
\hline Transport and communications & 155 & 161 & 65 & 33 & 54 \\
\hline Urban development and construction & 139 & 132 & 132 & 198 & 187 \\
\hline Economic development studies & 30 & 28 & 19 & 21 & 39 \\
\hline Social services & 3,183 & 3,253 & 3,839 & 4,996 & 6,198 \\
\hline Education & 2,276 & 2,511 & 2,981 & 3,906 & 4,896 \\
\hline Culture and sport & 52 & 71 & 64 & 82 & 135 \\
\hline Health & 526 & 532 & 696 & 822 & 1,009 \\
\hline Labor and social welfare & 61 & 76 & 57 & 143 & 107 \\
\hline Relief and rehabilitation & 268 & 63 & 40 & 42 & 50 \\
\hline Pension payments & 0 & 0 & 0 & 0 & 0 \\
\hline Interest and charges & 1,219 & 1,080 & 1,011 & 1,054 & 1,207 \\
\hline External assistance $3 /$ & 2,890 & 699 & 721 & 586 & 411 \\
\hline Miscellaneous & 230 & 525 & 325 & 67 & 76 \\
\hline Capital expenditure 4/ & 6,313 & 8,271 & 11,343 & 14,041 & 18,398 \\
\hline Economic development & 4,124 & 4,773 & 7,655 & 9,728 & 11,367 \\
\hline Agriculture & 929 & 1,876 & 2,889 & 3,359 & 3,410 \\
\hline Natural resources & 591 & 405 & 1,109 & 1,296 & 1,484 \\
\hline Mining and energy & 526 & 457 & 86 & 264 & 1,288 \\
\hline Trade, Industry and tourism & 40 & 112 & 277 & 176 & 234 \\
\hline Transport construction & 1,896 & 1,836 & 2,637 & 3,445 & 4,820 \\
\hline Transport and communications & 141 & 87 & 658 & 1,188 & 130 \\
\hline Financial agencies & 0 & 0 & 0 & 0 & 0 \\
\hline Social development & 1,745 & 2,233 & 3,290 & 3,796 & 5,998 \\
\hline Education & 1,017 & 1,666 & 1,896 & 2,479 & 3,514 \\
\hline Public health & 488 & 346 & 505 & 519 & 1,341 \\
\hline Urban development and housing & 210 & 165 & 828 & 714 & 1,038 \\
\hline Social welfare & 10 & 26 & 24 & 51 & 4 \\
\hline Culture and sport & 20 & 29 & 37 & 33 & 99 \\
\hline General services and compensation & 444 & 1,265 & 397 & 517 & 1,033 \\
\hline External assistance 5/ & 1,197 & 1,047 & 1,513 & 2,196 & 3,081 \\
\hline \multirow[t]{2}{*}{ Total expenditure } & 19,849 & 20,232 & 24,572 & 29,275 & 35,564 \\
\hline & \multicolumn{5}{|c|}{ (In percent of total expenditure) } \\
\hline Current expenditure & 68.2 & 59.1 & 53.8 & 52.0 & 48.3 \\
\hline General services 2/ & 23.6 & 24.9 & 23.7 & 22.3 & 19.9 \\
\hline Of which: defense & 11.8 & 12.1 & 11.9 & 10.3 & 8.4 \\
\hline Economic services & 6.7 & 6.7 & 6.2 & 6.9 & 6.2 \\
\hline Social services & 16.0 & 16.1 & 15.6 & 17.1 & 17.4 \\
\hline Interest and charges & 6.1 & 5.3 & 4.1 & 3.6 & 3.4 \\
\hline Other & 15.7 & 6.1 & 4.3 & 2.2 & 1.4 \\
\hline Capital expenditure 4/ & 31.8 & 40.9 & 46.2 & 48.0 & 51.7 \\
\hline Economic development & 20.8 & 23.6 & 31.2 & 33.2 & 32.0 \\
\hline Social development & 8.8 & 11.0 & 13.4 & 13.0 & 16.9 \\
\hline General services and compensation & 2.2 & 6.3 & 1.6 & 1.8 & 2.9 \\
\hline External assistance 5/ & 6.0 & 5.2 & 6.2 & 7.5 & 8.7 \\
\hline Total social spending (current and capital) & 43.3 & 50.3 & 57.0 & 60.1 & 62.9 \\
\hline Education & 16.6 & 20.6 & 19.8 & 21.8 & 23.6 \\
\hline Health & 5.1 & 4.3 & 4.9 & 4.6 & 6.6 \\
\hline \multirow[t]{2}{*}{ Other } & 21.6 & 25.3 & 32.3 & 33.7 & 32.6 \\
\hline & \multicolumn{5}{|c|}{ (In percent of GDP) } \\
\hline Current expenditure & 18.4 & 13.8 & 12.4 & 11.6 & 10.0 \\
\hline General services 2/ & 6.4 & 5.8 & 5.5 & 5.0 & 4.1 \\
\hline Of which: defense & 3.2 & 2.8 & 2.7 & 2.3 & 1.8 \\
\hline Economic services & 1.8 & 1.6 & 1.4 & 1.5 & 1.3 \\
\hline Social services & 4.3 & 3.8 & 3.6 & 3.8 & 3.6 \\
\hline Interest and charges & 1.7 & 1.2 & 0.9 & 0.8 & 0.7 \\
\hline Other & 4.2 & 1.4 & 1.0 & 0.5 & 0.3 \\
\hline Capital expenditure 4/ & 8.6 & 9.5 & 10.7 & 10.7 & 10.8 \\
\hline Economic development & 5.6 & 5.5 & 7.2 & 7.4 & 6.7 \\
\hline Social development & 2.4 & 2.6 & 3.1 & 2.9 & 3.5 \\
\hline General services and compensation & 0.6 & 1.5 & 0.4 & 0.4 & 0.6 \\
\hline External assistance $5 /$ & 1.6 & 1.2 & 1.4 & 1.7 & 1.8 \\
\hline Total social spending (current and capital) & 11.7 & 11.7 & 13.2 & 13.4 & 13.1 \\
\hline Education & 4.5 & 4.8 & 4.6 & 4.8 & 4.9 \\
\hline Health & 1.4 & 1.0 & 1.1 & 1.0 & 1.4 \\
\hline Other $6 /$ & 5.8 & 5.9 & 7.4 & 7.5 & 6.8 \\
\hline
\end{tabular}

Source: Ministry of Finance and Economic Development.

1/ Ethiopian fiscal year ending July 7.

$2 /$ Includes organs of state and judiciary.

$3 /$ Imputed value of goods and services provided through external grants, mainly aid in kind.

4/ Includes outlays other than investment proper, such as operating expenses of certain development agencies.

5/ Details across sectors unavailable. However, the 2003/04 estimate for external assistance is incorporated in sector estimates.

6 / Includes culture and sports, labor and social welfare, relief and rehabilitation, and urban development and housing. 
Table 13: Ethiopia. Summary of Regional Government Finances, 2002/03-2006/07 1/

\begin{tabular}{|c|c|c|c|c|c|}
\hline & $2002 / 03$ & 2003/04 & $2004 / 05$ & 2005/06 & 2006/07 \\
\hline & \multicolumn{4}{|c|}{ (In millions of birr) } & \\
\hline Total Revenue & 2,105 & 3,088 & 3,571 & 4,397 & 4,385 \\
\hline Tax Revenue & 1,565 & 2,165 & 2,686 & 2,854 & 3,234 \\
\hline Income and profits tax & 1,197 & 1,499 & 1,777 & 1,637 & 2,093 \\
\hline Land use fee & 132 & 300 & 361 & 640 & 300 \\
\hline Domestic sales and excise taxes & 161 & 265 & 438 & 448 & 667 \\
\hline Stamp duties & 75 & 101 & 110 & 129 & 174 \\
\hline Non tax revenue & 540 & 924 & 885 & 1,542 & 1,151 \\
\hline Total Expenditure & 6,234 & 7,717 & 8,849 & 11,233 & 13,387 \\
\hline Current Expenditure & 4,764 & 5,799 & 6,348 & 8,236 & 9,718 \\
\hline General Services & 1,418 & 1,722 & 1,882 & 2,389 & 2,825 \\
\hline Economic Services & 779 & 983 & 1,241 & 1,693 & 1,828 \\
\hline Social Services & 2,396 & 2,628 & 3,144 & 4,109 & 5,027 \\
\hline \multicolumn{6}{|l|}{ Pension payments } \\
\hline Miscellaneous & 171 & 466 & 81 & 45 & 39 \\
\hline Capital Expenditure & 1,470 & 1,918 & 2,501 & 2,997 & 3,668 \\
\hline Economic development & 791 & 978 & 1,161 & 1,466 & 1,621 \\
\hline Social development & 484 & 452 & 1,135 & 1,290 & 1,640 \\
\hline General development & 196 & 488 & 206 & 241 & 407 \\
\hline \multirow[t]{2}{*}{ Overall balance } & $-4,129$ & $-4,629$ & $-5,278$ & $-6,837$ & $-9,002$ \\
\hline & \multicolumn{5}{|c|}{ (In percent of GDP) } \\
\hline Total Revenue & 2.9 & 3.6 & 3.4 & 3.3 & 2.6 \\
\hline Tax Revenue & 2.1 & 2.5 & 2.5 & 2.2 & 1.9 \\
\hline Non tax revenue & 0.7 & 1.1 & 0.8 & 1.2 & 0.7 \\
\hline Total Expenditure & 8.5 & 8.9 & 8.3 & 8.5 & 7.8 \\
\hline Current Expenditure & 6.5 & 6.7 & 6.0 & 6.3 & 5.7 \\
\hline Of which: Social Services & 3.3 & 3.0 & 3.0 & 3.1 & 2.9 \\
\hline Capital Expenditure & 2.0 & 2.2 & 2.3 & 2.3 & 2.1 \\
\hline Of which: Social development & 0.7 & 0.5 & 1.1 & 1.0 & 1.0 \\
\hline \multirow[t]{2}{*}{ Overall balance } & -5.6 & -5.3 & -5.0 & -5.2 & -5.3 \\
\hline & \multicolumn{4}{|c|}{ (In percent of general government total) } & \\
\hline Total Revenue & 13.4 & 17.2 & 17.7 & 18.1 & 14.5 \\
\hline Tax Revenue & 19.0 & 19.8 & 21.7 & 20.2 & 18.6 \\
\hline Non tax revenue & 18.6 & 30.7 & 27.8 & 28.7 & 25.9 \\
\hline Total Expenditure & 30.4 & 37.6 & 35.7 & 38.3 & 37.6 \\
\hline Current Expenditure & 35.2 & 48.5 & 48.0 & 54.1 & 56.6 \\
\hline Of which: Social Services & 75.3 & 80.8 & 81.9 & 82.2 & 81.1 \\
\hline Capital Expenditure & 23.3 & 23.2 & 22.0 & 21.3 & 19.9 \\
\hline Of which: Social development & 27.7 & 20.3 & 34.5 & 34.0 & 27.3 \\
\hline
\end{tabular}

Source: Ministry of Finance and Economic Development.

$1 /$ Ethiopian fiscal year ending July 7. 
Table 14. Ethiopia: Monetary Survey, 2002/03-2006/07 1/2/

\begin{tabular}{|c|c|c|c|c|c|}
\hline & $2002 / 03$ & $2003 / 04$ & $2004 / 05$ & $2005 / 06$ & $2006 / 07$ \\
\hline & \multicolumn{5}{|c|}{ (In millions of birr, unless otherwise specified) } \\
\hline Foreign assets (net) & 11,290 & 14,000 & 13,896 & 13,251 & 13,927 \\
\hline National Bank of Ethiopia & 6,273 & 9,181 & 9,670 & 7,312 & 8,179 \\
\hline Assets & 8,003 & 11,665 & 13,477 & 9,389 & 11,807 \\
\hline Liabilities & 1,731 & 2,484 & 3,807 & 2,077 & 3,628 \\
\hline Commercial banks & 5,018 & 4,820 & 4,227 & 5,939 & 5,748 \\
\hline Assets & 6,731 & 6,713 & 6,133 & 7,684 & 7,719 \\
\hline Liabilities & 1,713 & 1,893 & 1,906 & 1,745 & 1,971 \\
\hline Domestic credit & 28,332 & 31,681 & 41,652 & 50,044 & 61,585 \\
\hline Claims on government (net) & 17,855 & 20,009 & 23,156 & 26,040 & 30,290 \\
\hline National Bank of Ethiopia & 9,804 & 9,383 & 19,071 & 18,804 & 24,065 \\
\hline Commercial banks & 8,052 & 10,627 & 4,085 & 7,236 & 6,225 \\
\hline Claims on nongovernment & 10,477 & 11,672 & 18,496 & 24,003 & 31,295 \\
\hline National Bank of Ethiopia & 114 & 54 & 0 & 0 & 0 \\
\hline Commercial banks & 10,364 & 11,618 & 18,496 & 24,003 & 31,295 \\
\hline Other items (net) & $-9,117$ & $-11,858$ & $-15,095$ & $-15,810$ & -18653 \\
\hline Broad money & 30,506 & 33,822 & 40,452 & 47,485 & 56,861 \\
\hline Money & 16,815 & 18,137 & 21,429 & 24,701 & 29,774 \\
\hline Currency outside banks & 7,726 & 7,891 & 10,063 & 11,360 & 13,704 \\
\hline Demand deposits & 9,089 & 10,246 & 11,366 & 13,341 & 16,070 \\
\hline Quasi-money & 13,691 & 15,684 & 19,023 & 22,784 & 27,087 \\
\hline Savings deposits & 12,529 & 14,447 & 17,404 & 20,688 & 23,759 \\
\hline \multirow[t]{2}{*}{ Time deposits } & 1,162 & 1,237 & 1,619 & 2,097 & 3,328 \\
\hline & \multicolumn{5}{|c|}{ (Annual percentage change with respect to beginning-period broad money) } \\
\hline Foreign assets (net) & 10.8 & 8.9 & -0.3 & -1.6 & 1.4 \\
\hline Domestic credit & 2.5 & 11.0 & 29.5 & 20.7 & 24.3 \\
\hline Claims on government (net) & 5.7 & 7.1 & 9.3 & 7.1 & 9.0 \\
\hline Claims on nongovernment & -3.2 & 3.9 & 20.2 & 13.6 & 15.4 \\
\hline Broad money & 10.4 & 10.9 & 19.6 & 17.4 & 19.7 \\
\hline Money & 4.9 & 4.3 & 9.7 & 8.1 & 10.7 \\
\hline \multirow[t]{2}{*}{ Quasi money } & 5.5 & 6.5 & 9.9 & 9.3 & 9.1 \\
\hline & \multicolumn{5}{|c|}{ (In millions of birr, unless otherwise specified) } \\
\hline \multicolumn{6}{|l|}{ Memorandum items: } \\
\hline Net domestic assets & 19,216 & 19,823 & 26,556 & 34,235 & 42,932 \\
\hline Reserve money & 12,664 & 13,168 & 22,902 & 20,570 & 26,211 \\
\hline Excess reserves (in percent of deposits) 3/ & 11.1 & 10.0 & 28.5 & 13.6 & 16.5 \\
\hline Quasi money/broad money (in percent) & 44.9 & 46.4 & 47.0 & 48.0 & 47.6 \\
\hline Velocity (GDP/broad money) & 2.4 & 2.6 & 2.6 & 2.8 & 3.0 \\
\hline
\end{tabular}

Source: National Bank of Ethiopia.

$1 /$ Ethiopian fiscal year ending July 7 .

2/ Net foreign assets exclude net claims on the Bank of Eritrea; from 2000/01, currency in circulation excludes old notes of Birr 1,036.5 million, redeemed in Eritrea and unclaimed in Ethiopia.

$3 /$ Including government deposits. 
Table 15. Ethiopia: Loan Portfolio of the Banking System, 2002/03-2006/07 1/ 2/

\begin{tabular}{|c|c|c|c|c|c|}
\hline & $2002 / 03$ & $2003 / 04$ & $2004 / 05$ & $2005 / 06$ & $2006 / 07$ \\
\hline & \multicolumn{5}{|c|}{ (In millions of birr) } \\
\hline Claims on the government (net) & 17,855 & 20,009 & 23,165 & 26,032 & 30290 \\
\hline Development Bank of Ethiopia advances & 114 & 54 & 0 & 0 & 0 \\
\hline Nongovernment & 13,118 & 13,881 & 17,705 & 21,025 & 25,374 \\
\hline Nonfinancial public enterprises $3 /$ & 785 & 1,572 & 2,447 & 2,525 & 2639 \\
\hline Financial public enterprises $4 /$ & 449 & 404 & 302 & 275 & 226 \\
\hline Cooperatives & 314 & 336 & 817 & 1,477 & 1748 \\
\hline Private sector & 11,570 & 11,568 & 14,139 & 16,748 & 20761 \\
\hline \multirow[t]{2}{*}{ Total } & 31,087 & 33,944 & 40,870 & 47,057 & 55,664 \\
\hline & \multicolumn{5}{|c|}{ (In percent of total) } \\
\hline Claims on the government (net) & 57.4 & 58.9 & 56.7 & 55.3 & 54.4 \\
\hline Development Bank of Ethiopia advances & 0.4 & 0.2 & 0.0 & 0.0 & 0.0 \\
\hline Nongovernment & 42.2 & 40.9 & 43.3 & 44.7 & 45.6 \\
\hline Nonfinancial public enterprises $3 /$ & 2.5 & 4.6 & 6.0 & 5.4 & 4.7 \\
\hline Financial public enterprises $4 /$ & 1.4 & 1.2 & 0.7 & 0.6 & 0.4 \\
\hline Cooperatives & 1.0 & 1.0 & 2.0 & 3.1 & 3.1 \\
\hline \multirow[t]{2}{*}{ Private sector } & 37.2 & 34.1 & 34.6 & 35.6 & 37.3 \\
\hline & \multicolumn{5}{|c|}{$\begin{array}{c}\text { (Percentage change with respect to total net claims of the } \\
\text { banking system at beginning of the fiscal year) }\end{array}$} \\
\hline Claims on the government (net) & 5.3 & 6.9 & 9.3 & 7.0 & 9.0 \\
\hline Development Bank of Ethiopia advances & -0.9 & -0.2 & -0.2 & 0.0 & 0.0 \\
\hline Nongovernment & -0.5 & 2.5 & 11.3 & 8.1 & 9.2 \\
\hline Nonfinancial public enterprises $3 /$ & -1.0 & 2.5 & 2.6 & 0.2 & 0.2 \\
\hline Financial public enterprises 4/ & -0.2 & -0.1 & -0.3 & -0.1 & -0.1 \\
\hline Cooperatives & 0.0 & 0.1 & 1.4 & 1.6 & 0.6 \\
\hline Private sector & 0.7 & 0.0 & 7.6 & 6.4 & 8.5 \\
\hline Total & 3.8 & 9.2 & 20.4 & 15.1 & 18.3 \\
\hline
\end{tabular}

Source: National Bank of Ethiopia.

$1 /$ Ethiopian fiscal year ending July 7.

2/ Includes provision for doubtful loans and loans to public banks, except the Development Bank of Ethiopia.

3/ Excludes equity investment.

4/ Excludes deposit claims on specialized banks. 
Table 16. Ethiopia: Sectoral Breakdown of Commercial Bank Claims on Nongovernment Sectors, 2002/03-2006/07 1/ 2/

\begin{tabular}{|c|c|c|c|c|c|}
\hline & $2002 / 03$ & $2003 / 04$ & $2004 / 05$ & $2005 / 06$ & $2006 / 07$ \\
\hline & \multicolumn{5}{|c|}{ (In millions of birr) } \\
\hline Agriculture & 1,203 & 1,162 & 2,018 & 3,418 & 4218 \\
\hline Manufacturing & 2,750 & 4,208 & 5,136 & 6,320 & 6997 \\
\hline Exports & 856 & 945 & 1,460 & 1,581 & 2334 \\
\hline Imports & 1,703 & 2,452 & 3,148 & 3,744 & 4166 \\
\hline Construction & 1,897 & 2,039 & 2,218 & 3,207 & 3791 \\
\hline Domestic trade and services & 2,880 & 3,302 & 3,864 & 5,271 & 6,908 \\
\hline Domestic trade & 1,948 & 2,193 & 2,660 & 3,346 & 3,821 \\
\hline Transport & 600 & 773 & 873 & 1,578 & 2,370 \\
\hline Hotel and tourism & 318 & 323 & 317 & 317 & 676 \\
\hline Other services & 14 & 14 & 14 & 31 & 41 \\
\hline Personal & 55 & 58 & 115 & 93 & 147 \\
\hline Others & 4,200 & 3,585 & 3,790 & 3,117 & 2,542 \\
\hline \multirow[t]{2}{*}{ Total reported } & 15,543 & 17,750 & 21,749 & 26,751 & 31,102 \\
\hline & \multicolumn{5}{|c|}{ (In percent of total) } \\
\hline Agriculture & 7.7 & 6.5 & 9.3 & 12.8 & 13.6 \\
\hline Manufacturing & 17.7 & 23.7 & 23.6 & 23.6 & 22.5 \\
\hline Exports & 5.5 & 5.3 & 6.7 & 5.9 & 7.5 \\
\hline Imports & 11.0 & 13.8 & 14.5 & 14.0 & 13.4 \\
\hline Construction & 12.2 & 11.5 & 10.2 & 12.0 & 12.2 \\
\hline Domestic trade and services & 18.5 & 18.6 & 17.8 & 19.7 & 22.2 \\
\hline Domestic trade & 12.5 & 12.4 & 12.2 & 12.5 & 12.3 \\
\hline Transport & 3.9 & 4.4 & 4.0 & 5.9 & 7.6 \\
\hline Hotel and tourism & 2.0 & 1.8 & 1.5 & 1.2 & 2.2 \\
\hline Other services & 0.1 & 0.1 & 0.1 & 0.1 & 0.1 \\
\hline Personal & 0.4 & 0.3 & 0.5 & 0.3 & 0.5 \\
\hline Others & 27.0 & 20.2 & 17.4 & 11.7 & 8.2 \\
\hline
\end{tabular}

Source: National Bank of Ethiopia.

1/ Data for each year pertain to June 30.

2/ Includes provision for doubtful loans and loans to public banks, except the Development Bank of Ethiopia. 
Table 17. Ethiopia: Commercial Bank Lending and Deposits, 2002/03-2006/07 1/2/

\begin{tabular}{|c|c|c|c|c|c|}
\hline & $2002 / 03$ & $2003 / 04$ & $2004 / 05$ & $2005 / 06$ & $2006 / 07$ \\
\hline Lending & \multicolumn{5}{|c|}{ (In millions of birr) } \\
\hline Central government & 10,275 & 13,743 & 8,337 & 13,220 & 13,161 \\
\hline Other sectors & 13,118 & 13,881 & 17,705 & 21,025 & 25,374 \\
\hline Nonfinancial public enterprises 3 & 785 & 1,572 & 2,447 & 2,525 & 2,639 \\
\hline Financial public enterprises $4 /$ & 449 & 404 & 302 & 275 & 226 \\
\hline Cooperatives & 314 & 336 & 817 & 1,477 & 1,748 \\
\hline Private sector & 11,570 & 11,568 & 14,139 & 16,748 & 20,761 \\
\hline \multirow[t]{2}{*}{ Total lending } & 23,393 & 27,624 & 26,042 & 34,245 & 38,535 \\
\hline & \multicolumn{5}{|c|}{ (In percent of total lending) } \\
\hline Central government & 43.9 & 49.8 & 32.0 & 38.6 & 34.2 \\
\hline Other sectors & 56.1 & 50.2 & 68.0 & 61.4 & 65.8 \\
\hline Nonfinancial public enterprises 3 & 3.4 & 5.7 & 9.4 & 7.4 & 6.8 \\
\hline Financial public enterprises 4/ & 1.9 & 1.5 & 1.2 & 0.8 & 0.6 \\
\hline Cooperatives & 1.3 & 1.2 & 3.1 & 4.3 & 4.5 \\
\hline Private sector & 49.5 & 41.9 & 54.3 & 48.9 & 53.9 \\
\hline Deposits & \multicolumn{5}{|c|}{ (In millions of birr) } \\
\hline Demand deposits & 13,396 & 15,621 & 18,060 & 21,587 & 25,598 \\
\hline Public enterprises & 3,637 & 3,735 & 4,062 & 4,234 & 5,259 \\
\hline Cooperatives & 432 & 600 & 789 & 926 & 1,137 \\
\hline Private sector & 4,392 & 5,186 & 6,341 & 7,813 & 9,450 \\
\hline Central government & 2,215 & 3,108 & 4,245 & 5,984 & 6,928 \\
\hline Other $5 /$ & 2,721 & 2,991 & 2,624 & 2,631 & 2,824 \\
\hline Savings deposits & 12,529 & 14,447 & 17,403 & 20,688 & 23,760 \\
\hline Public enterprises & 52 & 15 & 42 & 52 & 40 \\
\hline Cooperatives & 268 & 378 & 895 & 603 & 895 \\
\hline Private sector & 12,199 & 14,039 & 16,429 & 19,890 & 22,757 \\
\hline Other $5 /$ & 10 & 15 & 36 & 143 & 68 \\
\hline Time deposits & 1,170 & 1,245 & 1,627 & 2,105 & 3,336 \\
\hline Public enterprises & 113 & 138 & 165 & 210 & 504 \\
\hline Cooperatives & 33 & 52 & 47 & 38 & 70 \\
\hline Private sector & 556 & 616 & 794 & 888 & 968 \\
\hline Central government & 8 & 8 & 8 & 8 & 8 \\
\hline Other $5 /$ & 459 & 432 & 613 & 960 & 1,785 \\
\hline \multirow[t]{2}{*}{ Total deposits } & 27,095 & 31,313 & 37,090 & 44,380 & 52,694 \\
\hline & \multicolumn{5}{|c|}{ (In percent of deposits) } \\
\hline Demand deposits & 49.4 & 49.9 & 48.7 & 48.6 & 48.6 \\
\hline Savings deposits & 46.2 & 46.1 & 46.9 & 46.6 & 45.1 \\
\hline Time deposits & 4.3 & 4.0 & 4.4 & 4.7 & 6.3 \\
\hline
\end{tabular}

Source: National Bank of Ethiopia.

$1 /$ Ethiopian fiscal year ending July 7.

2/ Includes provision for doubtful loans and loans to public banks, except the

Development Bank of Ethiopia.

3/ Excludes equity investment.

4/ Excludes deposit claims on specialized banks.

$5 /$ Includes domestic financial agencies and nontransferable accounts of nonresidents. 
Table 18. Ethiopia: Reserve and Liquidity Position of Commercial Banks, 2002/03-2006/07

\begin{tabular}{lrrrrr}
\hline & $2002 / 03$ & $2003 / 04$ & $2004 / 05$ & $2005 / 06$ & $2006 / 07$ \\
& \multicolumn{4}{c}{ (In millions of birr, unless otherwise indicated) } \\
$\begin{array}{lrrrr}\text { Reserve requirement } \\
\text { Net deposits 2/ }\end{array}$ & 26,406 & 30,987 & 36,568 & 42,419 & 51850 \\
Reserve requirement 3/ & 1,320 & 1,549 & 1,828 & 2,121 & 2,593 \\
Actual reserves & 3,817 & 4,911 & 12,856 & 8,452 & 11,734 \\
Excess reserves & 2,497 & 3,362 & 11,027 & 6,331 & 9,142 \\
Actual reserve ratio (in percent) & 14.5 & 15.8 & 35.2 & 19.9 & 22.6 \\
Liquidity requirement & & & & & \\
Net current deposits 4/ & 25,258 & 29,759 & 34,948 & 40,331 & 48523 \\
Liquidity requirement 5/ & 3,789 & 4,464 & 5,242 & 6,050 & 7,278 \\
Actual liquid assets & 16,461 & 20,264 & 22,417 & 24,129 & 27,597 \\
Excess liquidity & 12,672 & 15,800 & 17,175 & 18,079 & 20,319 \\
Actual liquidity ratio (in percent) & 65.2 & 68.1 & 64.1 & 59.8 & 56.9 \\
\hline
\end{tabular}

Source: National Bank of Ethiopia.

1/ Data for each year pertain to June 30.

2/ Demand, savings, and time deposits, less uncleared checks paid, and less uncleared effects (foreign).

$3 /$ Five percent of net deposits.

4/ Net deposits, less deposits at notice (deposits that can be withdrawn after 30 days' notice).

5 / Fifteen percent of net current deposits. 
Table 19. Ethiopia: Structure of Interest Rates, Jun. 2003-March 2008 1/

\begin{tabular}{|c|c|c|c|c|c|c|c|c|c|c|c|c|c|c|c|c|c|c|}
\hline & \multirow{2}{*}{$\frac{2002}{\text { Jun. }}$} & \multirow{2}{*}{$\frac{2003}{\text { Jun. }}$} & \multirow{2}{*}{$\frac{2004}{\text { Jun. }}$} & \multicolumn{4}{|c|}{$2004 / 05$} & \multicolumn{4}{|c|}{$2005 / 06$} & \multicolumn{4}{|c|}{$2006 / 07$} & \multicolumn{3}{|c|}{$2007 / 08$} \\
\hline & & & & Sep. & Dec. & Mar. & Jun. & Sep. & Dec. & Mar. & Jun. & Sep. & Dec. & Mar. & Jun. & Sep. & Dec. & Mar. \\
\hline Deposit rates & \multicolumn{18}{|c|}{ (In percent per annum) } \\
\hline Savings deposits $2 /$ & 3.00 & 3.00 & 3.00 & 3.00 & 3.00 & 3.00 & 3.00 & 3.00 & 3.00 & 3.00 & 3.00 & 3.00 & 3.00 & 3.00 & 3.00 & 4.00 & 4.00 & 4.00 \\
\hline \multicolumn{19}{|l|}{ Time deposits $3 /$} \\
\hline Less than 12 months & 3.30 & 3.35 & 3.40 & 3.36 & 3.38 & 3.45 & 3.47 & 3.49 & 3.48 & 3.52 & 3.60 & 3.58 & 3.59 & 3.63 & 3.64 & 4.61 & 4.59 & 4.68 \\
\hline 1 year to less than 2 years & 3.51 & 3.62 & 3.64 & 3.60 & 3.64 & 3.69 & 3.71 & 3.74 & 3.73 & 3.79 & 4.01 & 4.01 & 4.02 & 4.07 & 4.11 & 5.16 & 5.13 & 5.25 \\
\hline 2 years and above & 3.57 & 3.82 & 3.84 & 3.81 & 3.86 & 3.92 & 3.94 & 3.99 & 3.99 & 4.06 & 4.30 & 4.40 & 4.42 & 4.44 & 4.49 & 5.52 & 5.47 & 5.61 \\
\hline \multicolumn{19}{|l|}{ Lending rates } \\
\hline Bank lending rates & 7.50 & 7.00 & 7.00 & 7.00 & 7.00 & 7.00 & 7.00 & 7.00 & 7.00 & 7.00 & 7.00 & 7.00 & 7.00 & 7.00 & 7.00 & 8.00 & 8.00 & 8.00 \\
\hline Minimum & 14.00 & 14.00 & 14.00 & 14.00 & 14.00 & 14.00 & 14.00 & 14.00 & 14.00 & 14.00 & 14.00 & 14.00 & 14.00 & 14.00 & 14.00 & 15.00 & 15.00 & 15.00 \\
\hline $\begin{array}{l}\text { Maximum } \\
\text { Average }\end{array}$ & 10.75 & 10.50 & 10.50 & 10.50 & 10.50 & 10.50 & 10.50 & 10.50 & 10.50 & 10.50 & 10.50 & 10.50 & 10.50 & 10.50 & 10.50 & 11.50 & 11.50 & 11.50 \\
\hline Treasury bill yield & 1.98 & 1.31 & 0.59 & 0.44 & 0.15 & 0.08 & 0.05 & 0.04 & 0.04 & 0.04 & 0.04 & 0.04 & 0.42 & 0.81 & 0.79 & 1.01 & 0.57 & 0.50 \\
\hline
\end{tabular}

Source: National Bank of Ethiopia.

1/ Data for end-year pertain to June 30.

2/ Minimum rate.

$3 /$ Weighted rate, but the weighting does not take in to account the structure of deposits. 
Table 20. Ethiopia: Exchange Rate Developments, 1990/91-2006/07 1/

\begin{tabular}{|c|c|c|c|c|c|}
\hline & $2002 / 03$ & $2003 / 04$ & $2004 / 05$ & $2005 / 06$ & $2006 / 07$ \\
\hline & \multicolumn{5}{|c|}{ (Period average $; 1996 / 97=100)$} \\
\hline \multicolumn{6}{|l|}{ Effective exchange rates } \\
\hline Nominal & 96.5 & 92.0 & 85.6 & 83.4 & 80.10 \\
\hline \multirow[t]{2}{*}{ Real } & 97.4 & 100.6 & 94.3 & 102.0 & 114.50 \\
\hline & \multicolumn{5}{|c|}{ (Annual percentage change; - depreciation) } \\
\hline Nominal & -6.4 & -4.6 & -6.9 & -2.7 & -3.9 \\
\hline Real & 14.2 & 3.3 & -6.3 & 8.2 & 12.3 \\
\hline Memorandum items: 2/ & \multicolumn{5}{|c|}{ (In units indicated) } \\
\hline Birr per U.S. dollar, end of period & 8.61 & 8.63 & 8.67 & 8.69 & 9.03 \\
\hline Birr per U.S. dollar, period average & 8.58 & 8.62 & 8.65 & 8.68 & 8.80 \\
\hline Birr per SDR, end of period $/ 3$ & 12.05 & 12.66 & 12.62 & 12.82 & 13.70 \\
\hline \multirow[t]{2}{*}{ Birr per SDR, period average } & 11.66 & 12.50 & 12.97 & 12.67 & 13.20 \\
\hline & \multicolumn{5}{|c|}{ (Annual percentage change; - depreciation) } \\
\hline Birr per U.S. dollar, end of period & -0.5 & -0.2 & -0.5 & -0.3 & -3.7 \\
\hline Birr per U.S. dollar, period average & -0.4 & -0.5 & -0.4 & -0.3 & -1.3 \\
\hline Birr per SDR, end of period & -5.4 & -4.8 & 0.3 & -1.6 & -6.4 \\
\hline Birr per SDR, period average & -6.9 & -6.7 & -3.6 & 2.4 & -4.0 \\
\hline
\end{tabular}

Sources: National Bank of Ethiopia and International Monetary Fund.

1/ Data pertain to the period July 1-June 30.

$2 /$ Since May 1993, marginal rates at foreign exchange auctions; since October 2001, rates in the interbank market.

3/ Data on Birr/SDR for 1989/90 - 2004/05, International Monetary Fund.

For 2005/06 and 2006/07 average of buying and selling rate of the Birr against the SDR was considered. 
Table 21. Ethiopia: Balance of Payments, 2002/03-2006/07 1/

\begin{tabular}{|c|c|c|c|c|c|}
\hline & $2002 / 03$ & $2003 / 04$ & $2004 / 05$ & $2005 / 06$ & $2006 / 07$ \\
\hline & \multicolumn{5}{|c|}{ (In millions of U.S. dollars) } \\
\hline Current account balance & -109 & -402 & -771 & $-1,387$ & -870 \\
\hline (excluding official transfers) & -709 & -969 & $-1,521$ & $-2,253$ & $-2,069$ \\
\hline Merchandise trade balance & $-1,374$ & $-1,986$ & $-2,786$ & $-3,592$ & $-3,939$ \\
\hline Exports & 483 & 600 & 847 & 1,000 & 1,187 \\
\hline Coffee & 165 & 223 & 335 & 354 & 424 \\
\hline Other & 318 & 377 & 512 & 646 & 763 \\
\hline Imports & 1,856 & 2,587 & 3,633 & 4,593 & 5,126 \\
\hline Fuel & 288 & 311 & 669 & 861 & 875 \\
\hline Cereals & 189 & 206 & 160 & 195 & 161 \\
\hline Aircraft & 17 & 126 & 139 & 68 & 38 \\
\hline Other & 1,362 & 1,944 & 2,666 & 3,470 & 4,053 \\
\hline Services (net) & 167 & 310 & 277 & 149 & 161 \\
\hline Income (net) & -66 & -64 & -36 & -38 & 14 \\
\hline Of which: Official interest payments & -72 & -62 & -51 & -62 & -35 \\
\hline Current transfers (net) & 1,164 & 1,338 & 1,773 & 2,096 & 2,895 \\
\hline Private transfers (net) & 564 & 771 & 1,023 & 1,229 & 1,696 \\
\hline Official transfers (net) & 600 & 567 & 750 & 866 & 1,199 \\
\hline Capital account balance & 417 & 512 & 609 & 530 & 697 \\
\hline Investment assets and liabilities (net) & 417 & 512 & 609 & 530 & 697 \\
\hline Official, medium and long term & 359 & 198 & 271 & 196 & 239 \\
\hline Disbursements & 443 & 298 & 344 & 343 & 345 \\
\hline Amortization & 84 & 100 & 73 & 148 & 106 \\
\hline Other public long term (net) 2/ & -4 & 190 & 193 & -7 & c \\
\hline \multicolumn{6}{|l|}{ Long-term private (net, including } \\
\hline foreign direct investment) & 123 & 150 & 150 & 365 & 482 \\
\hline Short-term public and private (net) & -61 & -26 & -6 & -24 & -24 \\
\hline Errors and omissions (net) & -34 & 117 & 66 & 507 & 170 \\
\hline Overall balance & 275 & 227 & -97 & -350 & -3 \\
\hline Financing & -275 & -227 & 97 & 350 & 3 \\
\hline Change in net foreign assets (increase -) & -345 & -308 & 12 & 217 & -85 \\
\hline Central bank & -236 & -334 & -44 & 284 & -39 \\
\hline Reserves (increase -) & -266 & -420 & -231 & 424 & -168 \\
\hline Liabilities & 30 & 86 & 187 & -140 & 129 \\
\hline Commercial banks & -108 & 25 & 56 & -67 & -46 \\
\hline Change in arrears & 1 & 0 & 0 & 0 & 0 \\
\hline \multirow[t]{2}{*}{ Debt relief $3 /$} & 69 & 82 & 84 & 133 & 88 \\
\hline & \multicolumn{5}{|c|}{ (In percent of GDP, unless otherwise indicated) } \\
\hline Current account balance & -1.3 & -4.0 & -6.3 & -9.1 & -4.5 \\
\hline (excluding official transfers) & -8.3 & -9.6 & -12.4 & -14.9 & -10.6 \\
\hline Merchandise trade balance & -16.1 & -19.8 & -22.6 & -23.7 & -20.3 \\
\hline Exports & 5.6 & 6.0 & 6.9 & 6.6 & 6.1 \\
\hline Imports & 21.7 & 25.7 & 29.5 & 30.3 & 26.4 \\
\hline (excluding external cereals/aid in kind) & 19.5 & 23.7 & 28.2 & 29.0 & 25.5 \\
\hline Capital account balance & 4.9 & 5.1 & 4.9 & 3.5 & 3.6 \\
\hline Overall balance & 3.2 & 2.3 & -0.8 & -2.3 & 0.0 \\
\hline \multicolumn{6}{|l|}{ Memorandum item: } \\
\hline GDP (in millions of U.S. dollars) & 8,558 & 10,054 & 12,307 & 15,168 & 19,436 \\
\hline \multicolumn{6}{|l|}{ Gross official reserve (End of period) } \\
\hline In million U.S. dollars & 931 & 1,352 & 1,581 & 1,158 & 1,326 \\
\hline In months of next year's imports & 3.7 & 3.8 & 3.5 & 2.2 & 2.0 \\
\hline
\end{tabular}

Sources: National Bank of Ethiopia, and Ministry of Finance and Economic Development

1/ Data for the period July 1-June 30

2/ Ethiopian Airlines and other public enterprises.

3/ Includes a Paris Club rescheduling agreement in 1997/98. 
Table 22. Ethiopia: Exports, 2002/03-2006/07 1/2/

\begin{tabular}{|c|c|c|c|c|c|}
\hline & $2002 / 03$ & 2003/04 & $2004 / 05$ & $2005 / 06$ & $2006 / 07$ \\
\hline & \multicolumn{5}{|c|}{$\begin{array}{l}\text { (Value in millions of U.S. dollars; volume in thousands of metric tons; and price } \\
\text { in U.S. dollars per thousand metric tons) }\end{array}$} \\
\hline Coffee & 165.2 & 223.6 & 335.2 & 354.3 & 424.2 \\
\hline Volume & 126.1 & 156.4 & 161.1 & 147.7 & 176.4 \\
\hline Price & 1.3 & 1.4 & 2.1 & 2.4 & 2.4 \\
\hline Pulses & 19.9 & 26.7 & 35.4 & 37.0 & 70.3 \\
\hline Volume & 66.2 & 77.8 & 121.7 & 110.4 & 158.8 \\
\hline Price & 0.3 & 0.3 & 0.3 & 0.3 & 0.4 \\
\hline Oilseeds & 46.5 & 83.6 & 125.0 & 211.4 & 187.4 \\
\hline Volume & 83.0 & 102.8 & 170.8 & 265.7 & 235.0 \\
\hline Price & 0.6 & 0.8 & 0.7 & 0.8 & 0.8 \\
\hline Sugar and molasses & 17.7 & 10.3 & $\ldots$ & $\ldots$ & $\ldots$ \\
\hline Volume & 77.0 & 16.0 & $\ldots$ & $\ldots$ & $\ldots$ \\
\hline Price & 0.2 & 0.6 & $\ldots$ & $\ldots$ & $\ldots$ \\
\hline Leather and leather products & 52.0 & 44.3 & 67.6 & 75.0 & 89.6 \\
\hline Volume & 10.5 & 13.1 & 15.6 & 15.4 & 15.8 \\
\hline Price & 5.0 & 3.4 & 4.3 & 4.9 & 5.7 \\
\hline Live animals & 0.5 & 2.1 & 12.8 & 27.6 & 36.8 \\
\hline Volume & 0.6 & 3.1 & 21.2 & 33.3 & 43.7 \\
\hline Price & 0.8 & 0.7 & 0.6 & 0.8 & 0.8 \\
\hline Meat, canned and frozen & 2.4 & 7.7 & 14.6 & 18.5 & 15.5 \\
\hline Volume & 1.7 & 4.0 & 7.3 & 8.0 & 5.9 \\
\hline Price & 1.4 & 1.9 & 2.0 & 2.3 & 2.6 \\
\hline Fruits and vegetables & 9.6 & 7.2 & 16.1 & 13.2 & 16.2 \\
\hline Volume & 25.3 & 29.4 & 37.9 & 34.8 & 40.9 \\
\hline Price & 0.4 & 0.2 & 0.4 & 0.4 & \\
\hline Flowers & $\ldots$ & $\ldots$ & 7.8 & 21.8 & 63.6 \\
\hline Volume & $\ldots$ & $\ldots$ & 2.5 & 6.3 & 13.6 \\
\hline Price & $\ldots$ & $\ldots$ & 3.1 & 3.5 & 4.7 \\
\hline Chat & 57.5 & 88.0 & 100.2 & 89.1 & 92.8 \\
\hline Volume & 11.0 & 13.8 & 19.4 & 22.3 & 22.7 \\
\hline Price & 5.2 & 6.4 & 5.2 & 4.0 & 4.1 \\
\hline Gold & 42.1 & 48.7 & 59.4 & 64.7 & 97.0 \\
\hline Volume & 5.0 & 6.2 & 6.0 & 5.0 & 5.6 \\
\hline Price & 8.4 & 7.9 & 9.9 & 13.0 & 17.4 \\
\hline Other exports $3 /$ & 69.4 & 58.3 & 73.2 & 87.8 & 93.6 \\
\hline Total exports & 482.8 & 600.5 & 847.4 & 1000.3 & 1187.1 \\
\hline \multirow[t]{2}{*}{ (excluding coffee) } & 317.5 & 377.0 & 512.0 & 646.0 & 762.9 \\
\hline & \multicolumn{5}{|c|}{ (In percent of GDP) } \\
\hline Coffee exports & 1.9 & 2.2 & 2.7 & 2.3 & 2.2 \\
\hline \multirow[t]{2}{*}{ Noncoffee exports } & 3.7 & 3.7 & 4.2 & 4.3 & 3.9 \\
\hline & \multicolumn{5}{|c|}{ (In percent of total exports) } \\
\hline Coffee & 34.2 & 37.2 & 39.6 & 35.4 & 35.7 \\
\hline Pulses & 4.1 & 4.4 & 4.2 & 3.7 & 5.9 \\
\hline Oilseeds & 9.6 & 13.9 & 14.8 & 21.1 & 15.8 \\
\hline Leather and leather products & 10.8 & 7.4 & 8.0 & 7.5 & 7.5 \\
\hline Chat & 11.9 & 14.7 & 11.8 & 8.9 & 7.8 \\
\hline Gold & 8.7 & 8.1 & 7.0 & 6.5 & 8.2 \\
\hline Other & 20.6 & 14.2 & 14.7 & 16.9 & 19.0 \\
\hline
\end{tabular}

Sources: National Bank of Ethiopia and Ethiopian Customs Authority.

1/ Data for the period July 1-June 30.

2/ Data based on customs records, except gold, for which data are reported by the National Bank of Ethiopia.

$3 /$ Includes textiles, essence oils, and spices. 
Table 23. Ethiopia: Imports, c.i.f., by End Use, 2002/03-2006/07 1/ 2/

\begin{tabular}{|c|c|c|c|c|c|}
\hline & $2002 / 03$ & $2003 / 04$ & $2004 / 05$ & $2005 / 06$ & $2006 / 07$ \\
\hline & \multicolumn{5}{|c|}{ (In millions of U.S. dollars) } \\
\hline Raw materials & 21.8 & 26.0 & 49.1 & 77.2 & 148.6 \\
\hline Semifinished goods & 274.6 & 435.2 & 664.7 & 821.6 & 765.5 \\
\hline Fuel & 287.7 & 310.5 & 668.7 & 860.5 & 875.1 \\
\hline Capital goods & 549.5 & 876.6 & $1,199.4$ & $1,452.8$ & $1,868.5$ \\
\hline Transport & 174.0 & 298.3 & 371.6 & 429.9 & 633.8 \\
\hline Of which: aircraft & 17.3 & 126.1 & 139.5 & 67.5 & 37.7 \\
\hline Agricultural & 5.9 & 10.8 & 24.4 & 38.7 & 33.0 \\
\hline Industrial & 369.6 & 567.5 & 803.4 & 984.2 & $1,201.7$ \\
\hline Consumer goods & 654.3 & 895.6 & 986.1 & $1,281.9$ & $1,317.0$ \\
\hline Durable & 183.6 & 294.6 & 337.3 & 415.7 & 520.7 \\
\hline Nondurable & 470.8 & 601.0 & 648.8 & 866.2 & 796.3 \\
\hline Of which: food & 231.7 & 269.0 & 247.2 & 332.7 & 258.5 \\
\hline Miscellaneous 3/ & 68.4 & 43.1 & 65.3 & 98.5 & 151.5 \\
\hline Total imports, c.i.f. & $1,856.4$ & $2,586.9$ & $3,633.3$ & $4,592.5$ & $5,126.2$ \\
\hline \multirow[t]{2}{*}{ (excluding fuel) } & $1,568.7$ & $2,276.4$ & $2,964.6$ & $3,732.0$ & $4,251.1$ \\
\hline & \multicolumn{5}{|c|}{ (In percent of GDP) } \\
\hline Nonfuel imports & 18.3 & 22.6 & 24.1 & 24.6 & 21.9 \\
\hline \multirow[t]{2}{*}{ Fuel imports } & 3.4 & 3.1 & 5.4 & 5.7 & 4.5 \\
\hline & \multicolumn{5}{|c|}{ (In percent of total) } \\
\hline Raw materials & 1.2 & 1.0 & 1.4 & 1.7 & 2.9 \\
\hline Semifinished goods & 14.8 & 16.8 & 18.3 & 17.9 & 14.9 \\
\hline Fuel & 15.5 & 12.0 & 18.4 & 18.7 & 17.1 \\
\hline Capital goods & 29.6 & 33.9 & 33.0 & 31.6 & 36.5 \\
\hline Consumer goods & 35.2 & 34.6 & 27.1 & 27.9 & 25.7 \\
\hline Miscellaneous & 3.7 & 1.7 & 1.8 & 2.1 & 3.0 \\
\hline
\end{tabular}

Sources: National Bank of Ethiopia and Ethiopian Customs Authority.

$1 /$ Data for the period July 1-June 30.

2/ Data based on customs records.

$3 /$ Includes military imports. 
Table 24. Ethiopia: Imports by Country of Origin, 2002/03-2006/07 1/

\begin{tabular}{|c|c|c|c|c|c|}
\hline & $2002 / 03$ & $2003 / 04$ & $2004 / 05$ & $2005 / 06$ & $2006 / 07$ \\
\hline & \multicolumn{5}{|c|}{ (In millions of birr) } \\
\hline European Union 2/ & 4,295 & 4,976 & 6,580 & 8,016 & 10,242 \\
\hline Belgium and Luxembourg & 393 & 335 & 592 & 918 & 900 \\
\hline France & 224 & 528 & 655 & 777 & 805 \\
\hline Germany & 691 & 836 & 1,044 & 1,498 & 1,656 \\
\hline Italy & 1,091 & 1,133 & 1,334 & 2,199 & 3,469 \\
\hline Netherlands & 424 & 534 & 460 & 621 & 620 \\
\hline United Kingdom & 717 & 767 & 860 & 873 & 777 \\
\hline Other & 755 & 844 & 1,637 & 1,131 & 2,015 \\
\hline Eastern Europe 2/ & 298 & 365 & 1,054 & 960 & 953 \\
\hline Russia & 216 & 218 & 373 & 286 & 433 \\
\hline Other & 82 & 146 & 681 & 674 & 521 \\
\hline Other Europe & 482 & 921 & 1,328 & 2,175 & 1,302 \\
\hline Turkey & 281 & 606 & 972 & 882 & 798 \\
\hline Other & 200 & 315 & 356 & 1,293 & 504 \\
\hline Total Europe & 5,074 & 6,263 & 8,963 & 11,034 & 12,497 \\
\hline Western Hemisphere & 1,192 & 2,813 & 3,763 & 3,769 & 2,619 \\
\hline Canada & 55 & 54 & 154 & 113 & 73 \\
\hline United States & 959 & 2,619 & 3,310 & 3,182 & 1,694 \\
\hline Other & 177 & 140 & 298 & 474 & 852 \\
\hline Asia and Middle East & 7,305 & 10,535 & 16,436 & 22,622 & 27,050 \\
\hline China & 1,481 & 2,303 & 3,525 & 5,008 & 7,647 \\
\hline India & 891 & 1,399 & 1,914 & 2,817 & 3,133 \\
\hline Japan & 1,043 & 1,014 & 1,052 & 1,285 & 2,778 \\
\hline Korea & 242 & 318 & 348 & 601 & 970 \\
\hline Saudi Arabia & 1,308 & 1,876 & 5,348 & 7,332 & 6,839 \\
\hline Other & 2,342 & 3,626 & 4,247 & 5,580 & 5,683 \\
\hline Africa & 1,242 & 1,342 & 2,084 & 2,366 & 2,821 \\
\hline Djibouti & 571 & 490 & 469 & 726 & 494 \\
\hline Kenya & 161 & 187 & 221 & 324 & 435 \\
\hline Other & 511 & 665 & 1,395 & 1,316 & 1,892 \\
\hline Australia & 47 & 42 & 82 & 56 & 87 \\
\hline Other (including unspecified) & 1,069 & 1,302 & 107 & 24 & 20 \\
\hline \multirow[t]{2}{*}{ Total imports, c.i.f. } & 15,930 & 22,298 & 31,434 & 39,870 & 45,094 \\
\hline & \multicolumn{5}{|c|}{ (In percent of total) } \\
\hline European Union & 27.0 & 22.3 & 20.9 & 20.1 & 22.7 \\
\hline Belgium and Luxembourg & 2.5 & 1.5 & 1.9 & 2.3 & 2.0 \\
\hline France & 1.4 & 2.4 & 2.1 & 1.9 & 1.8 \\
\hline Germany & 4.3 & 3.8 & 3.3 & 3.8 & 3.7 \\
\hline Italy & 6.9 & 5.1 & 4.2 & 5.5 & 7.7 \\
\hline Netherlands & 2.7 & 2.4 & 1.5 & 1.6 & 1.4 \\
\hline United Kingdom & 4.5 & 3.4 & 2.7 & 2.2 & 1.7 \\
\hline Other & 4.7 & 3.8 & 5.2 & 2.8 & 4.5 \\
\hline Eastern Europe & 1.9 & 1.6 & 3.4 & 2.4 & 2.1 \\
\hline Other Europe & 3.0 & 4.1 & 4.2 & 5.5 & 2.9 \\
\hline Total Europe & 31.9 & 28.1 & 28.5 & 27.7 & 27.7 \\
\hline Western Hemisphere & 7.5 & 12.6 & 12.0 & 9.5 & 5.8 \\
\hline Canada & 0.3 & 0.2 & 0.5 & 0.3 & 0.2 \\
\hline United States & 6.0 & 11.7 & 10.5 & 8.0 & 3.8 \\
\hline Other & 1.1 & 0.6 & 0.9 & 1.2 & 1.9 \\
\hline Asia and Middle East & 45.9 & 47.2 & 52.3 & 56.7 & 60.0 \\
\hline China & 9.3 & 10.3 & 11.2 & 12.6 & 17.0 \\
\hline India & 5.6 & 6.3 & 6.1 & 7.1 & 6.9 \\
\hline Japan & 6.5 & 4.5 & 3.3 & 3.2 & 6.2 \\
\hline Korea & 1.5 & 1.4 & 1.1 & 1.5 & 2.2 \\
\hline Saudi Arabia & 8.2 & 8.4 & 17.0 & 18.4 & 15.2 \\
\hline Other & 14.7 & 16.3 & 13.5 & 14.0 & 12.6 \\
\hline Africa & 7.8 & 6.0 & 6.6 & 5.9 & 6.3 \\
\hline Australia & 0.3 & 0.2 & 0.3 & 0.1 & 0.2 \\
\hline Other (including unspecified) & 6.7 & 5.8 & 0.3 & 0.1 & 0.0 \\
\hline
\end{tabular}

Sources: National Bank of Ethiopia and Ethiopian Customs Authority.

1/ Data for the period July 1-June 30.

2/ Hungary, Poland, Czech Republic and Slovakia are classified both under European Union and Eastern Europe. 
Table 25. Ethiopia: Exports by Country of Destination, 2001/02-2005/06 1/

\begin{tabular}{|c|c|c|c|c|c|}
\hline & $2001 / 02$ & $2002 / 03$ & $2003 / 04$ & $2004 / 05$ & $2005 / 06$ \\
\hline & \multicolumn{5}{|c|}{ (In millions of birr) } \\
\hline European Union 2/ & $1,386.3$ & $1,341.3$ & $1,593.8$ & $2,574.7$ & $2,511.7$ \\
\hline Belgium and Luxembourg & 118.8 & 76.6 & 169.6 & 375.4 & 234.0 \\
\hline France & 111.3 & 273.6 & 98.0 & 127.2 & 177.9 \\
\hline Germany & 437.9 & 352.9 & 560.3 & $1,068.7$ & 875.9 \\
\hline Italy & 391.9 & 183.2 & 307.0 & 384.8 & 473.9 \\
\hline Netherlands & 55.2 & 142.0 & 79.4 & 259.4 & 334.9 \\
\hline United Kingdom & 139.8 & 76.7 & 183.0 & 219.6 & 238.1 \\
\hline Other & 131.4 & 236.3 & 196.5 & 139.7 & 177.0 \\
\hline Eastern Europe 2/ & 29.0 & 13.6 & 11.2 & 39.1 & 34.9 \\
\hline Russia & 0.0 & 0.4 & 9.5 & 17.9 & 14.4 \\
\hline Other & 29.0 & 13.2 & 1.7 & 21.2 & 20.5 \\
\hline Other Europe & 235.5 & 93.0 & 628.3 & 486.6 & 715.8 \\
\hline Switzerland & 235.5 & 79.8 & 434.1 & 327.0 & 577.5 \\
\hline Other & $\ldots$ & 13.2 & 194.2 & 159.6 & 138.4 \\
\hline Total Europe & $1,650.8$ & $1,447.9$ & $2,233.3$ & $3,096.1$ & $3,256.80$ \\
\hline Western Hemisphere & 216.2 & 356.1 & 288.0 & 467.4 & 482.4 \\
\hline United States & 165.9 & 340.1 & 254.7 & 389.9 & 418.0 \\
\hline Other & 50.3 & 16.0 & 33.3 & 77.5 & 64.4 \\
\hline Asia and Middle East & $1,292.5$ & 854.2 & $1,570.8$ & $2,261.7$ & $3,387.9$ \\
\hline China & 91.0 & 22.5 & 109.6 & 362.8 & $1,166.7$ \\
\hline Israel & 149.0 & 201.0 & 169.8 & 179.9 & 178.0 \\
\hline Japan & 294.6 & 188.4 & 530.4 & 554.7 & 676.1 \\
\hline Saudi Arabia & 229.5 & 182.4 & 293.6 & 437.9 & 531.1 \\
\hline Other & 528.4 & 259.8 & 467.4 & 726.3 & 836.0 \\
\hline Africa & 411.9 & 792.1 & 910.7 & $1,033.8$ & $1,460.0$ \\
\hline Djibouti & 272.1 & 289.8 & 533.8 & 359.2 & 498.4 \\
\hline Kenya & 0.2 & 134.3 & 2.4 & 17.9 & 21.1 \\
\hline Other & 139.6 & 367.9 & 374.5 & 656.7 & 940.4 \\
\hline Australia & 7.9 & 4.4 & 11.7 & 12.0 & 28.5 \\
\hline Other (including unspecified) & 285.0 & 687.7 & 162.2 & 460.2 & 69.7 \\
\hline \multirow[t]{2}{*}{ Total exports, f.o.b. } & $3,864.3$ & $4,142.4$ & $5,176.7$ & $7,331.3$ & $8,685.4$ \\
\hline & \multicolumn{5}{|c|}{ (In percent of total) } \\
\hline European Union & 35.9 & 32.4 & 30.8 & 35.1 & 28.9 \\
\hline Belgium and Luxembourg & 3.1 & 1.8 & 3.3 & 5.1 & 2.7 \\
\hline France & 2.9 & 6.6 & 1.9 & 1.7 & 2.0 \\
\hline Germany & 11.3 & 8.5 & 10.8 & 14.6 & 10.1 \\
\hline Italy & 10.1 & 4.4 & 5.9 & 5.2 & 5.5 \\
\hline Netherlands & 1.4 & 3.4 & 1.5 & 3.5 & 3.9 \\
\hline United Kingdom & 3.6 & 1.9 & 3.5 & 3.0 & 2.7 \\
\hline Other & 3.4 & 5.7 & 3.8 & 1.9 & 2.0 \\
\hline Eastern Europe & 0.7 & 0.3 & 0.2 & 0.5 & 0.4 \\
\hline Other Europe & 6.1 & 2.2 & 12.1 & 6.6 & 8.2 \\
\hline Total Europe & 42.7 & 35.0 & 43.1 & 42.2 & 37.5 \\
\hline Western Hemisphere & 5.6 & 8.6 & 5.6 & 6.4 & 5.6 \\
\hline United States & 4.3 & 8.2 & 4.9 & 5.3 & 4.8 \\
\hline Other & 1.3 & 0.4 & 0.6 & 1.1 & 0.7 \\
\hline Asia and Middle East & 33.4 & 20.6 & 30.3 & 30.8 & 39.0 \\
\hline China & 2.4 & 0.5 & 2.1 & 4.9 & 13.4 \\
\hline Israel & 3.9 & 4.9 & 3.3 & 2.5 & 2.0 \\
\hline Japan & 7.6 & 4.5 & 10.2 & 7.6 & 7.8 \\
\hline Saudi Arabia & 5.9 & 4.4 & 5.7 & 6.0 & 6.1 \\
\hline Other & 13.7 & 6.3 & 9.0 & 9.9 & 9.6 \\
\hline Africa & 10.7 & 19.1 & 17.6 & 14.1 & 16.8 \\
\hline Australia & 0.2 & 0.1 & 0.2 & 0.2 & 0.3 \\
\hline Other (including unspecified) & 7.4 & 16.6 & 3.1 & 6.3 & 0.8 \\
\hline
\end{tabular}

Sources: National Bank of Ethiopia and Ethiopian Customs Authority.

1/ Includes reexports. Data pertain to the period July 1-June 30.

2/ Hungary, Poland, Czech Republic and Slovakia are classified both under European Union and

Eastern Europe. 
Table 26. Ethiopia: Public and Publicly Guaranteed External Debt Outstanding, 2002/03-2006/07

\begin{tabular}{|c|c|c|c|c|c|c|c|c|c|c|}
\hline & \multicolumn{2}{|c|}{$2002 / 03$} & \multicolumn{2}{|c|}{$2003 / 04$} & \multicolumn{2}{|c|}{$2004 / 05$} & \multicolumn{2}{|c|}{$2005 / 06$} & \multicolumn{2}{|c|}{$2006 / 07$} \\
\hline & Debt stock & $\begin{array}{c}\text { Of which: } \\
\text { arrears } 1 /\end{array}$ & Debt stock & $\begin{array}{l}\text { Of which: } \\
\text { arrears } 1 /\end{array}$ & Debt stock & $\begin{array}{c}\text { Of which: } \\
\text { arrears } 1 /\end{array}$ & Debt stock & $\begin{array}{l}\text { Of which: } \\
\text { arrears } 1 /\end{array}$ & Debt stock & $\begin{array}{l}\text { Of which: } \\
\text { arrears } 1 /\end{array}$ \\
\hline \multicolumn{11}{|c|}{ (In millions of U.S. dollars) } \\
\hline Multilateral & 4,249 & - & 4,670 & - & 4,881 & - & 4,878 & - & 1,130 & - \\
\hline IMF & 141 & - & 150 & - & 168 & - & - & - & - & - \\
\hline IDA & 2,939 & - & 3,258 & - & 3,362 & - & 3,513 & - & 621 & - \\
\hline AfDB/AfDF & 922 & - & 1,003 & - & 1,073 & - & 1,100 & - & 213 & - \\
\hline Other & 246 & - & 260 & - & 279 & - & 264 & - & 295 & - \\
\hline Official bilateral & 2,447 & 480 & 2,448 & - & 788 & 391 & 773 & 374 & 821 & 374 \\
\hline Paris Club & 1,880 & - & 1,886 & - & 229 & - & 336 & - & 371 & - \\
\hline Other official bilateral & 567 & 480 & 562 & - & 559 & 391 & 437 & 374 & 450 & 374 \\
\hline Commercial & 42 & 42 & 253 & - & 353 & 31 & 354 & 31 & 301 & 31 \\
\hline Total & 6,738 & 522 & 7,371 & - & 6,021 & 422 & 6,005 & 405 & 2,252 & 405 \\
\hline \multicolumn{11}{|c|}{ (In percent of GDP) } \\
\hline Multilateral & 49.6 & 0.0 & 46.4 & 0.0 & 39.7 & 0.0 & 32.2 & 0.0 & 5.8 & 0.0 \\
\hline Official bilateral & 28.6 & 5.6 & 24.3 & 0.0 & 6.4 & 3.2 & 5.1 & 2.5 & 4.2 & 1.9 \\
\hline Commercial & 0.5 & 0.5 & 2.5 & 0.0 & 2.9 & 0.3 & 2.3 & 0.2 & 1.5 & 0.2 \\
\hline Total & 78.7 & 6.1 & 73.3 & 0.0 & 48.9 & 3.4 & 39.6 & 2.7 & 11.6 & 2.1 \\
\hline \multicolumn{11}{|c|}{ (In percent of total) } \\
\hline Multilateral & 63.1 & 0.0 & 63.4 & 0.0 & 81.1 & 0.0 & 81.2 & 0.0 & 50.2 & 0.0 \\
\hline Official bilateral & 36.3 & 91.9 & 33.2 & 0.0 & 13.1 & 92.7 & 12.9 & 92.3 & 36.5 & 92.3 \\
\hline Commercial & 0.6 & 8.1 & 3.4 & 0.0 & 5.9 & 7.3 & 5.9 & 7.7 & 13.4 & 7.7 \\
\hline
\end{tabular}

Source: Ministry of Finance and Economic Development.

$1 /$ Includes debt under negotiation to ensure comparable treatment. 


\begin{tabular}{|c|c|c|c|c|}
\hline & $2003 / 04$ & $2004 / 05$ & $2005 / 06$ & $2006 / 07$ \\
\hline \multicolumn{5}{|l|}{ Capital adequacy } \\
\hline Capital to risk-weighted assets & 11.7 & 11.5 & 11.4 & 20.4 \\
\hline Capital to assets & 11.6 & 8.4 & 9.5 & 10.2 \\
\hline \multicolumn{5}{|l|}{ Asset composition and quality } \\
\hline Total loans and advances to total assets & 37.3 & 37.7 & 40.0 & 35.6 \\
\hline \multicolumn{5}{|l|}{ Sectoral distribution of loans to total loans } \\
\hline Manufacturing & 23.7 & 23.6 & 23.6 & 22.5 \\
\hline Construction & 11.5 & 10.2 & 12.0 & 12.2 \\
\hline Agriculture & 6.5 & 9.3 & 12.8 & 13.6 \\
\hline Transport & 4.4 & 4.0 & 5.9 & 7.6 \\
\hline Hotel and tourism & 1.8 & 1.5 & 1.2 & 2.2 \\
\hline Foreign exchange loans to total loans & - & - & - & \\
\hline Gross nonperforming loans to gross loans & 27.8 & 20.0 & 14.0 & 10.1 \\
\hline NPLs net of provisions to total capital & 83.1 & 63.7 & 45.4 & 7.6 \\
\hline Large exposures to total capital & 135.7 & 179.2 & 138.0 & 79.2 \\
\hline \multicolumn{5}{|l|}{ Earnings and profitability } \\
\hline $\mathrm{ROA}$ & 1.6 & 2.0 & 2.8 & 2.3 \\
\hline ROE & 21.8 & 32.1 & 41.1 & 28.4 \\
\hline Interest margin to gross income & 35.4 & 31.9 & 35.8 & 35.4 \\
\hline Noninterest expenses to gross income & 41.1 & 28.7 & 31.6 & 33.5 \\
\hline Personnel expenses to noninterest expenses & 30.3 & 42.6 & 43.1 & 43.8 \\
\hline Trading and fee income to total income & 43.3 & 47.5 & 45.9 & 46.4 \\
\hline Interest rate earned on loans and advances & 9.0 & 8.7 & 8.7 & 10.5 \\
\hline Interest rate paid on nonbank deposits & 3.9 & 3.9 & 4.0 & 3.6 \\
\hline Spread (lending minus deposit rates) & 5.1 & 4.8 & 4.7 & 6.9 \\
\hline \multicolumn{5}{|l|}{ Liquidity } \\
\hline Liquid assets to total assets & 52.7 & 47.3 & 44.6 & 40.4 \\
\hline Liquid assets to total short-term liabilities & 82.1 & 77.9 & 71.1 & 75.2 \\
\hline Total loans to customer deposits & 55.3 & 58.8 & 60.0 & 50.0 \\
\hline Foreign exchange liabilities to total liabilities & 0.7 & 0.9 & 0.9 & 0.6 \\
\hline
\end{tabular}

Sources: National Bank of Ethiopia; and IMF staff calculations. 
Ethiopia: Summary of the Tax System as of end-June 2008

\begin{tabular}{|c|c|c|c|c|c|}
\hline Tax & Nature of Tax & $\begin{array}{c}\text { Deductions and } \\
\text { Exemptions }\end{array}$ & \multicolumn{3}{|c|}{ Rates } \\
\hline \multicolumn{6}{|c|}{ 1. Taxes on income and profits. Income Tax Proclamation No. $286 / 2002$} \\
\hline $\begin{array}{l}1.1 \text { Income tax on } \\
\text { employment } \\
\text { Proclamation } \\
\text { No.286/2002. }\end{array}$ & $\begin{array}{l}\text { Tax on income from } \\
\text { employment, including, } \\
\text { without limitations, } \\
\text { salaries, wages, } \\
\text { allowances, directors' } \\
\text { fees, and other personal } \\
\text { emoluments. Tax is } \\
\text { withheld by employer. }\end{array}$ & $\begin{array}{l}\text { (1) The first Br } 150 \text { of monthly income } \\
\text { should be deducted prior to the } \\
\text { calculation of the tax. } \\
\text { (2) Income from employment received } \\
\text { by casual employees who are not } \\
\text { regularly employed provided that they } \\
\text { do not work for more than one month } \\
\text { for the same employer in any twelve } \\
\text { months. } \\
\text { (3) Pension contribution, provident } \\
\text { fund and all forms of retirement } \\
\text { benefits contributed by employers in } \\
\text { an amount that does not exceed } 15 \% \\
\text { of the monthly salary of the employee. } \\
\text { (4) Subject to reciprocity, income from } \\
\text { employment, received for services } \\
\text { rendered in the exercise of their } \\
\text { duties by: } \\
\text { (a) diplomatic and consular } \\
\text { representatives, and } \\
\text { (b) other persons employed in any } \\
\text { Embassy, Legation, Consulate or } \\
\text { Mission of a foreign state } \\
\text { performing state affairs, who are } \\
\text { national of that state and bearers } \\
\text { of diplomatic passports or who are } \\
\text { in accordance with international } \\
\text { usage or custom normally and } \\
\text { usually exempted from the } \\
\text { payment of income tax } \\
\text { (5) Income specifically exempted from } \\
\text { income tax by: } \\
\text { (a) a law in Ethiopia, unless } \\
\text { specifically amended or deleted by } \\
\text { this Proclamation; } \\
\text { (b) an international treaty; or } \\
\text { (b) the death of another person. } \\
\text { (c) an agreement made or approved } \\
\text { by the Minister. } \\
\text { (6) The Council of Ministers may by } \\
\text { regulations exempt any income } \\
\text { recognized as such by this } \\
\text { Proclamation for economic, } \\
\text { administrative or social reason. } \\
\text { (7) Payments made to a person as } \\
\text { compensation or a gratitude in } \\
\text { relation to: } \\
\text { (a) personal injuries suffered by that } \\
\text { (a) }\end{array}$ & 6 & $\begin{array}{l}\begin{array}{c}\text { Monthly taxable } \\
\text { income (birr) }\end{array} \\
151-650 \\
650-1,400 \\
1,400-2,350 \\
2,350-3,550 \\
3,550-5,000 \\
\text { Over } 5,000\end{array}$ & $\begin{array}{l}\text { as follows: } \\
\begin{array}{l}\text { Marginal tax } \\
\text { rate (\%) } \\
\\
10 \\
15 \\
20 \\
25 \\
30 \\
35\end{array}\end{array}$ \\
\hline
\end{tabular}


Ethiopia: Summary of the Tax System as of end-June 2008 (continued)

\begin{tabular}{|c|c|c|c|c|c|}
\hline Tax & Nature of Tax & $\begin{array}{l}\text { Deductions and } \\
\text { Exemptions }\end{array}$ & \multicolumn{3}{|c|}{ Rates } \\
\hline \multicolumn{6}{|c|}{ 1.2 Taxes on income from rental of buildings } \\
\hline $\begin{array}{l}1.2 .1 \text { Incorporated } \\
\text { business } \\
\text { Proclamation } \\
\text { No.286/2002. }\end{array}$ & $\begin{array}{l}\text { Tax on income derived } \\
\text { from the rent of houses } \\
\text { or office buildings, } \\
\text { manufacturing plants, } \\
\text { materials and goods, } \\
\text { etc. The tax is computed } \\
\text { on the basis of annual } \\
\text { rent income after } \\
\text { deducting allowable } \\
\text { expenses. }\end{array}$ & $\begin{array}{l}\text { The following amounts shall be } \\
\text { deducted from income in computing } \\
\text { taxable income: } \\
\text { (1) Taxes paid with respect to the } \\
\text { land and buildings being leased; } \\
\text { except income taxes; } \\
\text { (2) For taxpayers not maintaining } \\
\text { books of account, one fifth of the } \\
\text { gross income received as rent for } \\
\text { buildings furniture and equipment as } \\
\text { an allowance for repairs, } \\
\text { maintenance and depreciation of } \\
\text { such buildings, furniture and } \\
\text { equipment; } \\
\text { (3) For taxpayers maintaining books } \\
\text { of accounts, the expenses incurred in } \\
\text { earning, securing, and maintaining } \\
\text { rental income, to the extent that the } \\
\text { expenses can be proven by the } \\
\text { taxpayer and subject to the limitations } \\
\text { specified by this Proclamation; } \\
\text { deductible expenses include (but are } \\
\text { not limited to) the cost of lease (rent) } \\
\text { of land, repairs, maintenance, and } \\
\text { depreciation of buildings, furniture } \\
\text { and equipment in accordance with } \\
\text { Article } 23 \text { of this Proclamation as well } \\
\text { as interest on bank loans, insurance } \\
\text { premiums. } \\
\text { (4) The first Br } 1,800 \text { of annual } \\
\text { income should be deducted prior to } \\
\text { the calculation of the tax. }\end{array}$ & \multicolumn{3}{|c|}{ Thirty percent $(30 \%)$ of taxable income. } \\
\hline $\begin{array}{l}\text { 1.2.2 Unincorporated/ } \\
\text { Persons }\end{array}$ & As in 1.2.1 & As in 1.2.1 & & $\begin{array}{l}\text { Annual taxable } \\
\text { income (birr) }\end{array}$ & $\begin{array}{l}\text { Marginal tax } \\
\text { rate(\%) }\end{array}$ \\
\hline \multirow[t]{6}{*}{$\begin{array}{l}\text { Proclamation } \\
\text { No.286/2002. }\end{array}$} & & & 1 & $1,801-7,800$ & 10 \\
\hline & & & 2 & $7,800-16,800$ & 15 \\
\hline & & & 3 & $16,800-28,200$ & 20 \\
\hline & & & 4 & $28,200-42,600$ & 25 \\
\hline & & & 5 & $42,600-60,000$ & 30 \\
\hline & & & 6 & Over 60,000 & 35 \\
\hline
\end{tabular}


Ethiopia: Summary of the Tax System as of end-June 2008 (continued)

\begin{tabular}{|c|c|c|c|c|c|}
\hline Tax & Nature of Tax & $\begin{array}{c}\text { Deductions and } \\
\text { Exemptions }\end{array}$ & \multicolumn{3}{|c|}{ Rates } \\
\hline \multicolumn{6}{|c|}{ 1.3 Tax on business and other profits } \\
\hline $\begin{array}{l}\text { 1.3.1 Unincorporated } \\
\text { business } \\
\text { Proclamation No. } \\
286 / 2002 \text {. }\end{array}$ & $\begin{array}{l}\text { Tax imposed on the } \\
\text { taxable business income } \\
\text { realized from } \\
\text { entrepreneurial activity. } \\
\text { The taxable income is } \\
\text { determined per tax } \\
\text { period on the basis of } \\
\text { the profit and loss } \\
\text { account or income } \\
\text { statement, which shall } \\
\text { be drawn in compliance } \\
\text { with the Generally } \\
\text { Accepted Accounting } \\
\text { Standards, subject to the } \\
\text { provisions of this } \\
\text { Proclamation and } \\
\text { directives issued by the } \\
\text { Tax Authority. }\end{array}$ & $\begin{array}{l}\text { The first Br } 1,800 \text { of annual income } \\
\text { should be deducted prior to the } \\
\text { calculation of the tax. } \\
\text { The following categories of income } \\
\text { shall be exempt of business income } \\
\text { tax: } \\
\text { (1) Awards for adapted or suggested } \\
\text { innovations and cost saving } \\
\text { measures } \\
\text { (2) Public awards for outstanding } \\
\text { performance tax of any field } \\
\text { (3) Income specifically exempted } \\
\text { from income tax by the law in force in } \\
\text { Ethiopia, by international treaty or by } \\
\text { an agreement made or approved by } \\
\text { the Minister. } \\
\text { (4) The revenue obtained by: } \\
\text { (a) the Federal, Regional and } \\
\text { Local Governments of Ethiopia; } \\
\text { (b) the National Bank of Ethiopia } \\
\text { from activities that are } \\
\text { incidental to their operations } \\
\text { shall be exempt from tax on } \\
\text { business income. }\end{array}$ & $\begin{array}{l}1 \\
2 \\
3 \\
4 \\
5 \\
6\end{array}$ & $\begin{array}{l}\begin{array}{l}\text { Annual taxable } \\
\text { income (birr) }\end{array} \\
1,801-7,800 \\
7,800-16,800 \\
16,800-28,200 \\
28,200-42,600 \\
42,600-60,000 \\
\text { Over } 60,001\end{array}$ & $\begin{array}{r}\text { Marginal tax } \\
\text { rate (\%) } \\
\\
\\
10 \\
15 \\
\\
20\end{array}$ \\
\hline $\begin{array}{l}\text { 1.3.2 Incorporated } \\
\text { business Proclamation } \\
\text { No. } 286 / 2002 \text {. }\end{array}$ & As in 1.3.1 & As in 1.3.1 & 30 percent of tax & able income. & \\
\hline
\end{tabular}


Ethiopia: Summary of the Tax System as of end-June 2008 (continued)

\begin{tabular}{|c|c|c|c|}
\hline Tax & Nature of Tax & $\begin{array}{l}\text { Deductions } \\
\text { and } \\
\text { Exemptions }\end{array}$ & Rates \\
\hline \multicolumn{4}{|l|}{ 1.4 Taxes on Other Incomes } \\
\hline $\begin{array}{l}\text { 1.4.1 Tax on income } \\
\text { from mining activities } \\
\text { (Royalties) }\end{array}$ & $\begin{array}{l}\text { Tax collected from Royalty. Where Royalty } \\
\text { means a payment of any kind received as a } \\
\text { consideration for the use of, or the right to } \\
\text { use, any copyright of literary, artistic or } \\
\text { scientific work, including cinematography } \\
\text { films, and films or tapes for radio or television } \\
\text { broadcasting, any patent, trade work, design } \\
\text { or model, plan, secret formula or process, or } \\
\text { for the use or for the right to use of any } \\
\text { industrial, commercial or scientific } \\
\text { equipment, or for information concerning } \\
\text { industrial, commercial or scientific } \\
\text { experience. } \\
\text { The withholding Agent who effects payment } \\
\text { shall withhold the foregoing tax and account } \\
\text { to the Tax Authority with in the time limit set } \\
\text { out in this Proclamation. }\end{array}$ & $\begin{array}{l}\text { Artisan mining is } \\
\text { exempted. }\end{array}$ & 5 Percent on income earned from Royalty. \\
\hline $\begin{array}{l}\text { 1.4.2 Income tax on } \\
\text { Income from Rendering } \\
\text { of Technical Services to } \\
\text { organizations in Ethiopia } \\
\text { by non-resident persons } \\
\text { or organizations. }\end{array}$ & $\begin{array}{l}\text { Tax liable on all payments obtained in } \\
\text { consideration of any kind of technical } \\
\text { services rendered outside Ethiopia to } \\
\text { resident persons in any form. Where } \\
\text { "Technical service" means any kind of expert } \\
\text { advise or technological service rendered. }\end{array}$ & None. & 10 Percent on the payment made. \\
\hline $\begin{array}{l}\text { 1.4.3 Tax on Income } \\
\text { from Games of Chance }\end{array}$ & $\begin{array}{l}\text { Tax on income derived from winning at } \\
\text { games of chance (for example, lotteries, } \\
\text { tombolas, and other similar activities) }\end{array}$ & $\begin{array}{l}\text { Tax is not paid on } \\
\text { winnings of less than } \\
100 \text { Birr. }\end{array}$ & 15 Percent \\
\hline 1.4.4 Dividends & $\begin{array}{l}\text { Tax on income derived from dividends from a } \\
\text { share company or withdrawals of profits from } \\
\text { a private limited company. The withholding } \\
\text { Agent shall withhold or collect the tax and } \\
\text { account to the Tax Authority. }\end{array}$ & None. & 10 Percent \\
\hline $\begin{array}{l}\text { 1.4.5 Tax on income } \\
\text { from Rental of Property }\end{array}$ & $\begin{array}{l}\text { Tax on income derived from the causal rental } \\
\text { of property (including any land, building, or } \\
\text { movable asset) not related to a business } \\
\text { activity taxable under } 1.2 \text { above. }\end{array}$ & None. & 15 Percent on the annual gross income \\
\hline $\begin{array}{l}\text { 1.4.6 Tax on Interest } \\
\text { Income on Deposits }\end{array}$ & $\begin{array}{l}\text { Tax on income derived from interest on } \\
\text { deposits. The payer shall withhold the tax } \\
\text { and account to the Tax Authority. }\end{array}$ & None. & 5 Percent \\
\hline $\begin{array}{l}\text { 1.4.7 Tax on the Gain on } \\
\text { the Transfer of Certain } \\
\text { Investment Property }\end{array}$ & $\begin{array}{l}\text { Income tax on gains obtained from the } \\
\text { transfer (sale or gift) of property. }\end{array}$ & $\begin{array}{l}\text { Gains obtained from the } \\
\text { transfer of building held } \\
\text { for residence is exempt. }\end{array}$ & $\begin{array}{l}\text { The following rates are levied: } \\
\text { (a) } 15 \text { Percent for building } \\
\text { held for business, factory, and } \\
\text { office. } \\
\text { (b) } 30 \text { Percent for shares of } \\
\text { companies. }\end{array}$ \\
\hline $\begin{array}{l}1.5 \text { Rural land and } \\
\text { agricultural activities } \\
\text { income tax }\end{array}$ & & & Varies from region to region. \\
\hline
\end{tabular}


Ethiopia: Summary of the Tax System as of end-June 2008 (continued)

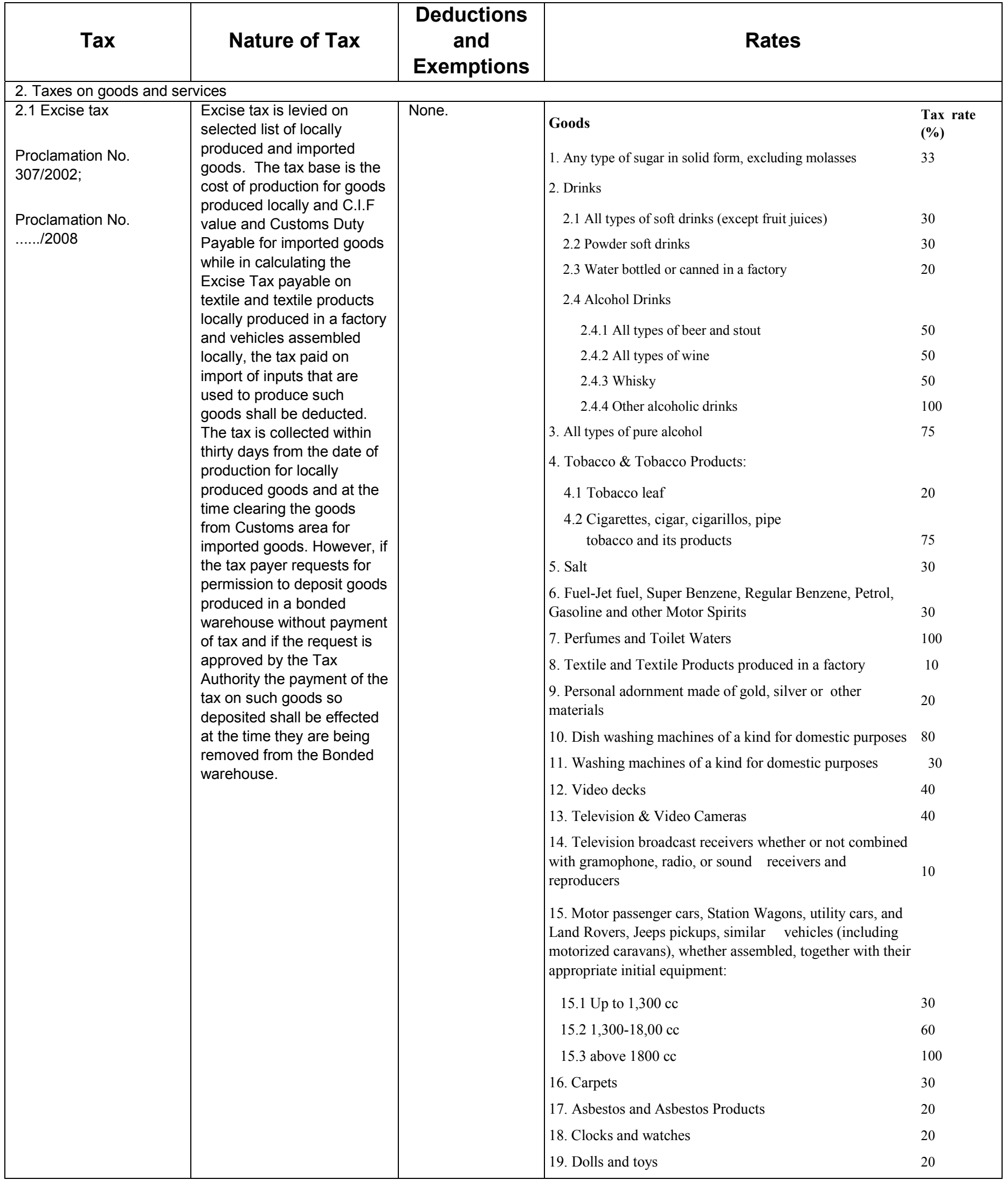


Ethiopia: Summary of the Tax System as of end-June 2008 (continued)

\begin{tabular}{|c|c|c|c|}
\hline Tax & Nature of Tax & Deductions and Exemptions & Rates \\
\hline $\begin{array}{l}2.2 \text { Value Added Tax } \\
\text { (VAT) on Goods and } \\
\text { Services } \\
\text { Proclamation } \\
\text { No.285/2002. }\end{array}$ & $\begin{array}{l}\text { Levied on locally } \\
\text { produced goods at the } \\
\text { manufacturing level or } \\
\text { on imported goods at the } \\
\text { import gate. The bases } \\
\text { for taxation are the } \\
\text { producer's wholesale } \\
\text { price plus excise tax for } \\
\text { local goods or the c.i.f. } \\
\text { value plus customs duty } \\
\text { and excise tax for } \\
\text { imports. There is a } \\
\text { refund for sales tax paid } \\
\text { on raw materials used in } \\
\text { the production of local } \\
\text { goods, except for pure } \\
\text { alcohol used as raw } \\
\text { material. The tax is } \\
\text { payable monthly within } \\
\text { three to five days of the } \\
\text { end of the month. For } \\
\text { imported goods, the tax } \\
\text { is collected at the same } \\
\text { time as the customs } \\
\text { duty. }\end{array}$ & 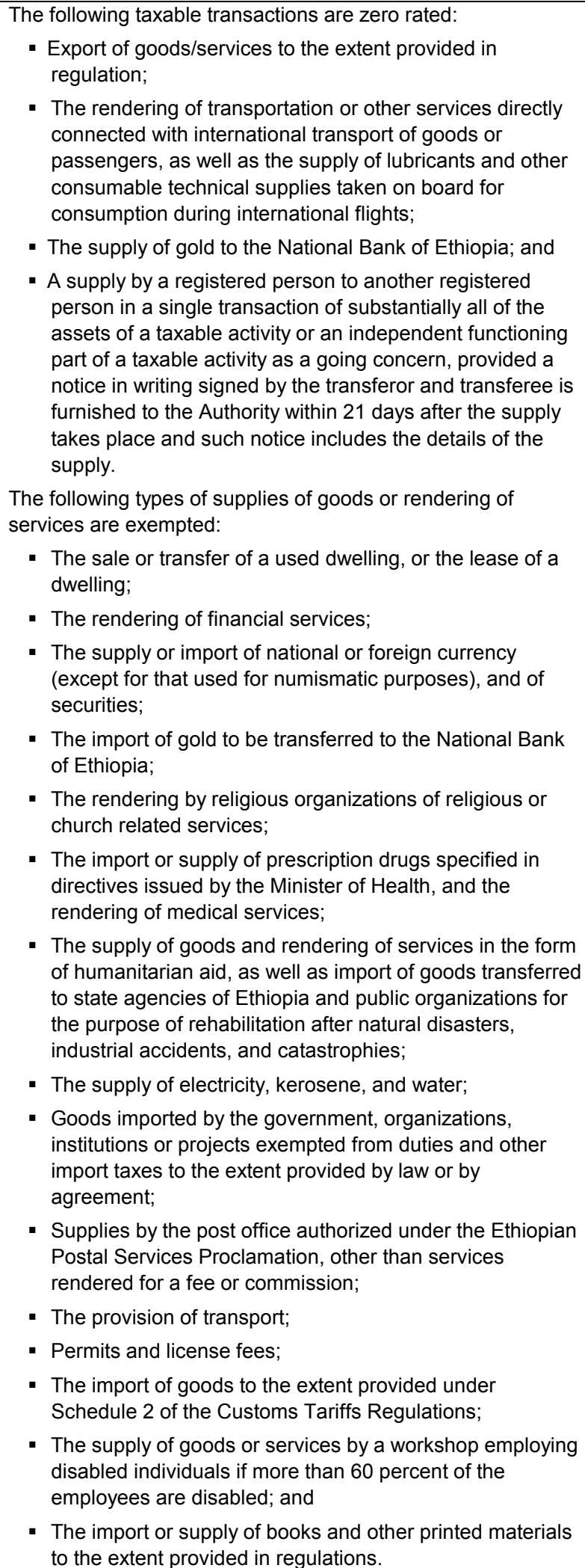 & $\begin{array}{l}15 \% \text { (Fifteen percent) on } \\
\text { each transacted sale of } \\
\text { goods and services. }\end{array}$ \\
\hline
\end{tabular}


Ethiopia: Summary of the Tax System as of end-June 2008 (continued)

\begin{tabular}{|c|c|c|c|}
\hline Tax & Nature of Tax & Deductions and Exemptions & Rates \\
\hline \multicolumn{4}{|l|}{$\begin{array}{l}\text { 2.3 Turn Over Tax } \\
\text { (ToT) }\end{array}$} \\
\hline $\begin{array}{l}\text { 2.3.1 ToT on } \\
\text { Goods sold locally }\end{array}$ & $\begin{array}{l}\text { The tax is levied on the } \\
\text { gross receipts of goods } \\
\text { supplied locally }\end{array}$ & $\begin{array}{l}\text { - Transfer of a dwelling used for a minimum of } \\
\text { two years; } \\
\text { - The supply of prescription drugs specified in } \\
\text { directive issued by the relevant agency, and } \\
\text { the rendering of medical services; } \\
\text { - The supply of goods and rendering of } \\
\text { services in the form of humanitarian aid } \\
\text { - The supply of electricity, kerosene, and } \\
\text { water; } \\
\text { - The supply of goods or services by workshop } \\
\text { employing disabled individuals if more than } \\
60 \% \text { of the employees are disabled; and } \\
\text { - The supply of books }\end{array}$ & $\begin{array}{l}\text { Two percent }(2 \%) \text { on annual turnover of } \\
\text { the goods supplier. }\end{array}$ \\
\hline $\begin{array}{l}\text { 2.3.2 ToT on } \\
\text { Services rendered } \\
\text { locally }\end{array}$ & $\begin{array}{l}\text { The tax is levied on the } \\
\text { services rendered locally }\end{array}$ & $\begin{array}{l}\text { - The lease of a dwelling; } \\
\text { - Rendering of financial services } \\
\text { - The supply of national currency (except for } \\
\text { that used for numismatic purposes) and } \\
\text { securities; } \\
\text { - The rendering by religious organizations of } \\
\text { religious or other related services; } \\
\text { - The rendering of educational services } \\
\text { provided by educational institutions, as well } \\
\text { as child care services for children at pre- } \\
\text { school institutions; } \\
\text { - The provision of transport; and } \\
\text { - Permits and license fees }\end{array}$ & $\begin{array}{l}\text { (a) Two percent ( } 2 \%) \text { on Contractors, grain } \\
\text { mills, tractors and combine-harvesters. } \\
\text { (b) Ten percent }(10 \%) \text { on other services }\end{array}$ \\
\hline \multicolumn{4}{|l|}{$\begin{array}{l}\text { 3. Tax on } \\
\text { International Trade }\end{array}$} \\
\hline \multicolumn{4}{|l|}{$\begin{array}{l}3.1 \text { Taxes on } \\
\text { imports }\end{array}$} \\
\hline $\begin{array}{l}3.1 .1 \text { Customs } \\
\text { Duty } \\
\text { Tariff Regulation } \\
80 / 2002 . \\
\text { Tariff Regulation } \\
\ldots . . / 2008 .\end{array}$ & $\begin{array}{l}\text { The customs tariff applies } \\
\text { to all imports. Items are } \\
\text { classified according to a } \\
\text { schedule of } 97 \text { chapters, } \\
\text { based on the Harmonized } \\
\text { System of Tariffs } \\
\text { Classification Code. }\end{array}$ & $\begin{array}{l}\text { Diplomatic and consular missions, personal } \\
\text { effects, grants and gifts to Ethiopia, firefighting } \\
\text { instruments and appliances, trade samples, } \\
\text { defense and public security equipment, materials } \\
\text { and equipment for the handicapped, and sheath } \\
\text { contraceptives are exempted. Exemptions and } \\
\text { concessions are granted to certain organizations } \\
\text { and items. }\end{array}$ & $\begin{array}{l}\text { Ad valorem duty on imports consisting of six } \\
\text { rates, ranging from } 0 \text { to } 35 \text { percent, as } \\
\text { follows: } \\
\text { Imports } \\
\text { Tax rate (\%) } \\
\begin{array}{l}\text { Raw materials, capital } \\
\text { goods, pharmaceuticals, and } \\
\text { chemicals }\end{array} \\
\begin{array}{l}\text { Durable and nondurable } \\
\text { consumer goods }\end{array} \\
\begin{array}{l}\text { Luxuries and goods that can } \\
\text { be produced locally }\end{array}\end{array}$ \\
\hline $\begin{array}{l}3.1 .2 \text { Import excise } \\
\text { taxes }\end{array}$ & $\begin{array}{l}\text { Levied on selected } \\
\text { imported goods. }\end{array}$ & None. & As specified in (2.1) \\
\hline $\begin{array}{l}\text { 3.1.3 Import Value } \\
\text { Added Tax }\end{array}$ & $\begin{array}{l}\text { Sales tax levied on imports; } \\
\text { the basis for taxation is the } \\
\text { c.i.f. value plus the } \\
\text { customs duty and excise } \\
\text { tax paid. }\end{array}$ & See items in (2.2) for exemptions. & As specified in (2.2) \\
\hline
\end{tabular}


Ethiopia: Summary of the Tax System as of end-June 2008 (concluded)

\begin{tabular}{|c|c|c|c|c|}
\hline Tax & Nature of Tax & $\begin{array}{l}\text { Deductions and } \\
\text { Exemptions }\end{array}$ & \multicolumn{2}{|c|}{ Rates } \\
\hline 3.2 Taxes on exports & $\begin{array}{l}\text { All export duties and taxes are } \\
\text { abolished except on export of raw } \\
\text { and semi-processed hides \& skins; } \\
\text { the objective being to encourage } \\
\text { export of finished goods of hides and } \\
\text { skin. } \\
\text { A duty draw back and duty free } \\
\text { import schemes have been } \\
\text { introduced. Under the duty drawback } \\
\text { scheme, goods produced for export, } \\
\text { re-exporting or raw materials } \\
\text { imported upon payment of duties are } \\
\text { subject to refund of the duty paid. } \\
\text { Ninety-five percent of the duty drawn } \\
\text { back is refunded for raw materials or } \\
\text { commodities if re-exported and } 100 \\
\text { percent if exported after being } \\
\text { processed or used for packing or } \\
\text { containing. Under the duty free } \\
\text { import scheme, persons or } \\
\text { organizations wholly engaged for } \\
\text { supplying their products to foreign } \\
\text { markets are allowed a duty free } \\
\text { purchase of local or imported raw } \\
\text { material for their production, and the } \\
\text { product should be exported within } \\
\text { one year of the purchase of such } \\
\text { raw materials. }\end{array}$ & None. & 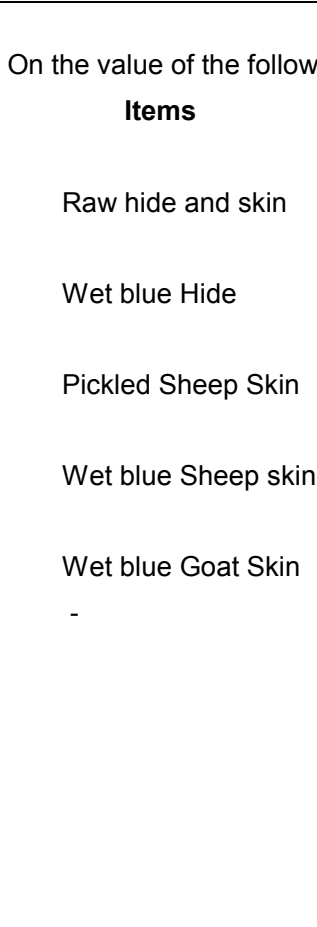 & $\begin{array}{l}\text { ms: } \\
\text { Rates } \\
15 \\
20 \\
10 \\
5 \\
5\end{array}$ \\
\hline 4. Other taxes & & & & \\
\hline $\begin{array}{l}.1 \text { Stamp duties } \\
\text { Decree No. } \\
26 / 1975 \text {, as } \\
\text { amended by } \\
\text { Proclamation } \\
\text { No.110/1998. }\end{array}$ & $\begin{array}{l}\text { Obligatory use of stamped paper for } \\
\text { many legal documents, such as } \\
\text { contracts or papers in court } \\
\text { proceedings; requirement that } \\
\text { stamps be affixed to other } \\
\text { documents, such as property titles, } \\
\text { power of attorney, notary acts, etc. }\end{array}$ & $\begin{array}{l}\text { Stamp duties are not levied on } \\
\text { documents where the Ethiopian } \\
\text { government, foreign embassies, } \\
\text { consulates, etc.., would be the } \\
\text { party subject to duty. }\end{array}$ & \multicolumn{2}{|c|}{$\begin{array}{l}\text { Varies according to the type of document } \\
\text { and value of transaction. }\end{array}$} \\
\hline $\begin{array}{l}\text { 4.2 Withholding tax: } \\
\text { Current payments } \\
\text { of income tax at } \\
\text { time goods are } \\
\text { imported. } \\
\text { Proclamation } \\
227 / 2001\end{array}$ & $\begin{array}{l}\text { Tax collected at the time of import of } \\
\text { goods for commercial use, where the } \\
\text { collected tax is treated as tax } \\
\text { withhold that is creditable against the } \\
\text { tax payer. }\end{array}$ & & \multicolumn{2}{|l|}{ Three percent } \\
\hline $\begin{array}{l}4.3 \text { Income tax: } \\
\text { Withholding of } \\
\text { income tax on } \\
\text { payments. Proclam } \\
\text { ation } 227 / 2001\end{array}$ & $\begin{array}{l}\text { Category "A" taxpayers, government } \\
\text { agencies, private nonprofit institution } \\
\text { and NGOs withhold income tax on } \\
\text { payments made to persons within } \\
\text { categories specified, in the } \\
\text { regulation No.75/2001. }\end{array}$ & & \multicolumn{2}{|l|}{ Two Percent } \\
\hline
\end{tabular}

